\title{
6j symbols for the modular double, quantum hyperbolic geometry, and supersymmetric gauge theories
}

\author{
J. Teschner and G. S. Vartanov \\ DESY Theory, Notkestr. 85, 22603 Hamburg, Germany
}

\begin{abstract}
We revisit the definition of the $6 j$ symbols from the modular double of $\mathcal{U}_{q}(\mathfrak{s l}(2, \mathbb{R}))$, referred to as b- $6 j$ symbols. Our new results are (i) the identification of particularly natural normalization conditions, and (ii) new integral representations for this object. This is used to briefly discuss possible applications to quantum hyperbolic geometry, and to the study of certain supersymmetric gauge theories. We show, in particular, that the b-6 $j$ symbol has leading semiclassical asymptotics given by the volume of a non-ideal tetrahedron. We furthermore observe a close relation with the problem to quantize natural Darboux coordinates for moduli spaces of flat connections on Riemann surfaces related to the Fenchel-Nielsen coordinates. Our new integral representations finally indicate a possible interpretation of the b- $6 j$ symbols as partition functions of non-abelian three-dimensional $\mathcal{N}=2$ supersymmetric gauge theories.
\end{abstract}

\section{Introduction}

Analogs of the Racah-Wigner $6 j$-symbols coming from the study of a non-compact quantum group have been introduced in [PT1]. The quantum group in question is related to $\mathcal{U}_{q}(\mathfrak{s l}(2, \mathbb{R}))$ and is often referred to as the modular double of $\mathcal{U}_{q}(\mathfrak{s l}(2, \mathbb{R}))$. The $6 j$-symbols of this quantum group, which will be called b-6 $j$ symbols, play an important role for the harmonic analysis of the modular double [ [PT2], quantum Liouville theory [T01] and quantum Teichmüller theory [T03]. The terminology b- $6 j$ symbol is partly motivated by the fact that it is useful to parameterize the deformation parameter $q$ of $\mathcal{U}_{q}(\mathfrak{s l}(2, \mathbb{R}))$ in terms of a parameter $b$ as $q=e^{\pi \mathrm{i} b^{2}}$.

However, the precise definition of the b- $6 j$ depends on the normalization of the ClebschGordan maps. Similar normalization issues arise in Liouville theory and in quantum Teichmüller theory. In the case of Liouville theory it is related to the issue to fix normalizations for bases in the space of conformal blocks. In quantum Teichmüller theory it is related to the 
precise definition of the representations in which a maximal commuting set of geodesic length operators is diagonal. The normalizations chosen in the references above were somewhat adhoc. One of our first goals in this paper is to discuss natural ways to fix this issue.

We will show that there exist very natural normalizations which also appear to be very natural from the point of view of Liouville- and the quantum Teichmüller theory. In the latter context, one of the normalizations defining our b- $6 j$ symbols will be shown to define a quantization of the Fenchel-Nielsen coordinates. Somewhat strikingly, we will find that the b- $6 j$ symbols defined in this way exactly reproduce the hyperbolic volume of a non-ideal tetrahedron with given dihedral angles in the classical limit $b \rightarrow 0$. This strongly suggests that Turaev-Viro type [TuVi] state-sum models built from the b- $6 j$ symbols are related to three-dimensional quantum gravity with negative cosmological constant, which can be seen as an analog of earlier observations for the cases of zero $[\overline{\mathrm{PR}}]$ and positive cosmological constants $[\mathrm{MT}]$, respectively. The $\mathrm{b}-6 j$ symbols are also natural building blocks for combinatorial approaches to the quantization of $S L(2, \mathbb{R})$-Chern-Simons theory or of its complexification.

One of our main technical results will be new integral representations for the b- $6 j$ symbols. One of them strongly resembles the formulae for the usual $6 j$ symbols. The new integral representations will be obtained from the formula for the b-6j symbols obtained in [PT2] by a sequence of nontrivial integral transformations that follow from an identity satisfied by Spiridonov's elliptic hypergeometric integrals [S01, S03] (for a review see [S08]) in certain limits. We will point out that one of these integral representations admits an interpretation as a partition function for a non-abelian three-dimensional supersymmetric gauge theory. This, and the relations to three-dimensional Chern-Simons theories mentioned above suggest that the b- $6 j$ symbols could play a key role in the currently investigated program to identify correspondences between three-dimensional supersymmetric gauge theories and noncompact Chern-Simons theories on suitable three-manifolds [TY, DiGu, DiGG].

\section{Racah-Wigner 6j symbols for the modular double}

\subsection{Self-dual representations of $\mathcal{U}_{q}(\mathfrak{s l}(2, \mathbb{R}))$ and the modular double}

We will be considering the Hopf-algebra $\mathcal{U}_{q}(\mathfrak{s l}(2, \mathbb{R}))$ which has generators $E, F$ and $K$ subject to the usual relations. This algebra has a one-parameter family of representations $\mathcal{P}_{\alpha}$

$$
\begin{aligned}
& \mathrm{E}_{\alpha} \equiv \pi_{\alpha}(E):=e^{+\pi b \times} \frac{\cosh \pi b(\mathrm{p}-s)}{\sin \pi b^{2}} e^{+\pi b \times}, \\
& \mathrm{F}_{\alpha} \equiv \pi_{\alpha}(F):=e^{-\pi b \times} \frac{\cosh \pi b(\mathrm{p}+s)}{\sin \pi b^{2}} e^{-\pi b \times}
\end{aligned}
$$


where $\mathrm{p}$ and $\mathrm{x}$ are operators acting on functions $f(x)$ as $\mathrm{p} f(x)=(2 \pi i)^{-1} \frac{\partial}{\partial x} f(x)$ and $\times f(x)=$ $x f(x)$, respectively. In the definitions (2.1) we are parameterizing $q$ as $q=e^{\pi \mathrm{i} b^{2}}$, and write the parameter $\alpha$ as $\alpha=Q / 2+\mathrm{i}$. There is a maximal dense subspace $\mathcal{P}_{\alpha} \subset L^{2}(\mathbb{R})$ on which all polynomials formed out of $\mathrm{E}_{\alpha}, \mathrm{F}_{\alpha}$ and $\mathrm{K}_{\alpha}$ are well-defined [BT2, Appendix B].

These representations are distinguished by a remarkable self-duality property: They are automatically representations of the quantum group $\mathcal{U}_{\tilde{q}}(\mathfrak{s l}(2, \mathbb{R}))$, where $\tilde{q}=e^{\pi \mathrm{i} / b^{2}}$ if $q=e^{\pi \mathrm{i} b^{2}}$. These representations are generated from operators $\tilde{\mathrm{E}}_{\alpha}, \tilde{\mathrm{F}}_{\alpha}$ and $\tilde{\mathrm{K}}_{\alpha}$ which are defined by formulae obtained from those in (2.1) by replacing $b \rightarrow b^{-1}$. The subspace $\mathcal{P}_{\alpha}$ is simultaneously a maximal domain for the polynomial functions of $\tilde{\mathrm{E}}_{\alpha}, \tilde{\mathrm{F}}_{\alpha}$ and $\tilde{\mathrm{K}}_{\alpha}[\mathrm{BT2}$, Appendix B].

This phenomenon was observed independently in [PT1] and in [F99]. It is closely related to the fact that $\mathrm{E}_{\alpha}, \mathrm{F}_{\alpha}$ and $\mathrm{K}_{\alpha}$ are positive self-adjoint generators which allows one to construct $\tilde{\mathrm{E}}_{\alpha}, \tilde{\mathrm{F}}_{\alpha}$ and $\tilde{\mathrm{K}}_{\alpha}$ as $\mathrm{E}_{\alpha}^{1 / b^{2}} \mathrm{~F}_{\alpha}^{1 / b^{2}}, \mathrm{~K}_{\alpha}^{1 / b^{2}}[\mathrm{BT} 1]$.

It was proposed in [PT1, BT1] to construct a noncompact quantum group which has as complete set of tempered representations the self-dual representations $\mathcal{P}_{\alpha}$. It's gradually becoming clear how to realize this suggestion precisely. Relevant steps in this direction were taken in [BT1] by defining co-product, R-operator and Haar-measure of such a quantum group. Further important progress in this direction was recently made in [Ip] $]$. Following [F99], we will in the following call this noncompact quantum group the modular double of $\mathcal{U}_{q}(\mathfrak{s l}(2, \mathbb{R}))$.

\subsection{Normalized Clebsch-Gordan coefficients for the modular double}

The Clebsch-Gordan maps $C_{\alpha_{2}, \alpha_{1}}^{\alpha_{3}}: \mathcal{P}_{\alpha_{2}} \otimes \mathcal{P}_{\alpha_{1}} \rightarrow \mathcal{P}_{\alpha_{3}}$ were constructed in [PT2]. The defining intertwining property is

$$
\mathrm{C}_{\alpha_{2}, \alpha_{1}}^{\alpha_{3}} \cdot\left(\pi_{\alpha_{2}} \otimes \pi_{\alpha_{1}}\right)(\Delta(X))=\pi_{\alpha_{3}} \cdot \mathrm{C}_{\alpha_{2}, \alpha_{1}}^{\alpha_{3}}
$$

In [PT2] it was found that the $\mathrm{C}_{\alpha_{2}, \alpha_{1}}^{\alpha_{3}}$ can be represented as integral operators of the form

$$
\left(C_{\alpha_{2}, \alpha_{1}}^{\alpha_{3}} \psi\right)\left(x_{3}\right)=\int_{\mathbb{R}^{2}} d x_{1} d x_{2}\left(\begin{array}{c|cc}
\alpha_{3} \\
x_{3}
\end{array} \mid \begin{array}{lll}
\alpha_{2} & \alpha_{1} \\
x_{1}
\end{array}\right)_{b} \psi\left(x_{2}, x_{1}\right),
$$

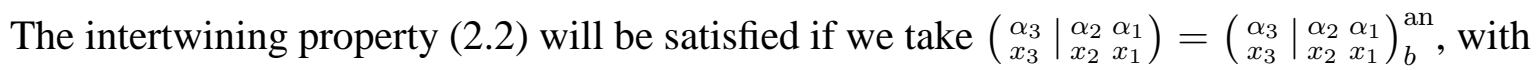

$$
\begin{aligned}
& \left(\begin{array}{c}
\alpha_{3} \\
x_{3}
\end{array} \mid \begin{array}{cc}
\alpha_{2} & \alpha_{1} \\
x_{2} & x_{1}
\end{array}\right)_{b}^{\text {an }}=e^{-\pi \mathrm{i}\left(\Delta_{\alpha_{3}}-\Delta_{\alpha_{1}}-\Delta_{\alpha_{2}}\right) / 2} D_{-\frac{\mathrm{i}}{2}\left(\alpha_{1}+\alpha_{2}+\alpha_{3}-Q\right)}\left(x_{2}-x_{1}-\mathrm{i} \frac{\alpha_{3}}{2}\right) \\
& \times D_{-\frac{i}{2}\left(Q+\alpha_{2}-\alpha_{3}-\alpha_{1}\right)}\left(x_{2}-x_{3}-\mathrm{i} \frac{\alpha_{1}}{2}\right) D_{-\frac{i}{2}\left(Q+\alpha_{1}-\alpha_{3}-\alpha_{2}\right)}\left(x_{3}-x_{1}-\mathrm{i} \frac{\alpha_{2}}{2}\right) \text {. }
\end{aligned}
$$

In (2.4) we are using the notations $\Delta_{\alpha}=\alpha(Q-\alpha)$ with $Q=b+b^{-1}$ and

$$
D_{\mathrm{i} \alpha}(x)=\frac{S_{b}(Q / 2-\mathrm{i} x+\alpha)}{S_{b}(Q / 2-\mathrm{i} x-\alpha)} .
$$


$S_{b}(x)$ is the so-called double Sine-function which is closely related to the functions called quantum dilogarithm in [FK2] hyperbolic gamma function in [ [Ru], and quantum exponential function in [Wo]. Definition and relevant properties are recalled in Appendix $\mathrm{A}$

One should note, however, that our definition of the $3 j$ coefficients (2.4) is not canonical, we might equally well use $\left(\begin{array}{c|cc}\alpha_{3} & \alpha_{2} & \alpha_{1} \\ x_{3} & x_{2} & x_{1}\end{array}\right)_{b}^{\prime}$ in (2.3), with

$$
\left(\begin{array}{c|cc}
\alpha_{3} & \alpha_{2} & \alpha_{1} \\
x_{3} & x_{2} & x_{1}
\end{array}\right)_{b}^{\prime}:=M\left(\alpha_{3}, \alpha_{2}, \alpha_{1}\right)\left(\begin{array}{c|cc}
\alpha_{3} & \begin{array}{cc}
\alpha_{2} & \alpha_{1} \\
x_{3}
\end{array} \\
x_{2} & x_{1}
\end{array}\right)_{b}^{\text {an }}
$$

This will satisfy (2.2) for arbitrary functions $M\left(\alpha_{3}, \alpha_{2}, \alpha_{1}\right)$. A natural choice for $M\left(\alpha_{3}, \alpha_{2}, \alpha_{1}\right)$ can be determined by requiring the Weyl-invariance of the Clebsch-Gordan maps. In order to formulate this requirement, we will need the intertwining operator $\mathrm{R}_{\alpha}: \mathcal{P}_{\alpha} \rightarrow \mathcal{P}_{Q-\alpha}$ which can be represented explicitly as integral operator [PT2]

$$
\left(\mathrm{R}_{\alpha} f\right)(x):=S_{b}(2 \alpha) \int_{\mathbb{R}} d x^{\prime} D_{-\mathrm{i} \alpha}\left(x-x^{\prime}\right) f(x) .
$$

We may now require that

$$
\begin{aligned}
& \mathrm{C}_{\alpha_{2}, \alpha_{1}}^{\alpha_{3}} \cdot\left(1 \otimes \mathrm{R}_{Q-\alpha_{1}}\right)=\mathrm{C}_{\alpha_{2}, Q-\alpha_{1}}^{\alpha_{3}}, \\
& \mathrm{C}_{\alpha_{2}, \alpha_{1}}^{\alpha_{3}} \cdot\left(\mathrm{R}_{Q-\alpha_{2}} \otimes 1\right)=\mathrm{C}_{Q-\alpha_{2}, \alpha_{1}}^{\alpha_{3}},
\end{aligned} \quad \mathrm{R}_{\alpha_{3}} \cdot \mathrm{C}_{\alpha_{2}, \alpha_{1}}^{\alpha_{3}}=\mathrm{C}_{\alpha_{2}, \alpha_{1}}^{Q-\alpha_{3}} .
$$

We claim that (2.8) is satisfied if we choose $M\left(\alpha_{3}, \alpha_{2}, \alpha_{1}\right)$ as

$$
\begin{aligned}
& M\left(\alpha_{3}, \alpha_{2}, \alpha_{1}\right)= \\
& =\left(S_{b}\left(2 Q-\alpha_{1}-\alpha_{2}-\alpha_{3}\right) S_{b}\left(Q-\alpha_{1}-\alpha_{2}+\alpha_{3}\right) S_{b}\left(\alpha_{1}+\alpha_{3}-\alpha_{2}\right) S_{b}\left(\alpha_{2}+\alpha_{3}-\alpha_{1}\right)\right)^{-\frac{1}{2}}
\end{aligned}
$$

To prove this claim let us consider, for example, the first of the equations in (2.8), which would follow from the identity

$$
S_{b}\left(2 \bar{\alpha}_{1}\right) \int_{\mathbb{R}} d x_{1}^{\prime}\left(\begin{array}{c|cc}
\alpha_{3} & \alpha_{2} & \alpha_{1} \\
x_{3} & x_{2} & x_{1}^{\prime}
\end{array}\right)_{b}^{\mathrm{an}} D_{-\mathrm{i} \bar{\alpha}_{1}}\left(x_{1}^{\prime}-x_{1}\right)=\xi\left(\begin{array}{c|cc}
\alpha_{3} & \alpha_{2} & \bar{\alpha}_{1} \\
x_{3} & x_{2} & x_{1}
\end{array}\right)_{b}^{\text {an }},
$$

where we use abbreviation $\bar{\alpha}=Q-\alpha$ and $\xi=S_{b}\left(\alpha_{2}+\alpha_{3}-\alpha_{1}\right) S_{b}\left(2 Q-\alpha_{1}-\alpha_{2}-\alpha_{3}\right)$. This identity can easily be rewritten in the form [BT1, Equation (A.34)] in which it is recognized as the famous star-triangle relation, see e.g. [BMS1]. Proofs can be found in [K2, $\mathrm{V}]$. It can also be derived easily from the so-called elliptic beta-integral [S01] following the strategy discussed in Appendix B.

We will denote the Clebsch-Gordan coefficients defined by (2.6) with function $M\left(\alpha_{3}, \alpha_{2}, \alpha_{1}\right)$ given in (2.9) as $\left(\begin{array}{l|ll}\alpha_{3} \\ x_{3}\end{array} \mid \begin{array}{ll}\alpha_{2} & \alpha_{1} \\ x_{2} & x_{1}\end{array}\right)_{b}$. We would like to stress that both $\left(\begin{array}{lll}\alpha_{3} & \alpha_{2} & \alpha_{1} \\ x_{3} & x_{2} & x_{1}\end{array}\right)_{b}^{\text {an }}$ and $\left(\begin{array}{lll}\alpha_{3} \\ x_{3}\end{array} \mid \begin{array}{ll}\alpha_{2} & \alpha_{1} \\ x_{2} & x_{1}\end{array}\right)_{b}$ have their virtues. While $\left(\begin{array}{l|ll}\alpha_{3} & \alpha_{2} & \alpha_{1} \\ x_{3} & x_{2} & x_{1}\end{array}\right)_{b}$ has more natural symmetry properties, the virtue of $\left(\begin{array}{l|ll}\alpha_{3} & \alpha_{2} & \alpha_{1} \\ x_{3} & x_{2} & x_{1}\end{array}\right)_{b}^{\text {an }}$ is to have nice analytic properties in all of its variables. 


\subsection{Normalized b-6j symbols for the modular double}

The composition of Clebsch-Gordan maps allows us to define two natural families of projection operators

$$
\begin{aligned}
& \left({ }^{s} C_{\alpha_{3}, \alpha_{2}, \alpha_{1}}^{\alpha_{4}}\left(\alpha_{s}\right) \Psi\right)\left(x_{4}\right)=\int_{\mathbb{R}^{3}} d x_{1} d x_{2} d x_{3} \mathcal{E}_{\alpha_{s}}^{(s)}(A \mid X) \psi\left(x_{3}, x_{2}, x_{1}\right), \\
& \left({ }^{t} C_{\alpha_{3}, \alpha_{2}, \alpha_{1}}^{\alpha_{4}}\left(\alpha_{t}\right) \Psi\right)\left(x_{4}\right)=\int_{\mathbb{R}^{3}} d x_{1} d x_{2} d x_{3} \mathcal{E}_{\alpha_{t}}^{(t)}(A \mid X) \psi\left(x_{3}, x_{2}, x_{1}\right),
\end{aligned}
$$

with integral kernels $\mathcal{E}_{\alpha_{s}}^{(s)}(A \mid X)$ and $\mathcal{E}_{\alpha_{t}}^{(t)}(A \mid X)$ given as

$$
\begin{aligned}
& \mathcal{E}_{\alpha_{s}}^{(s)}(A \mid X)=\int d x_{s}\left(\begin{array}{c|cc}
\alpha_{4} & \alpha_{3} & \alpha_{s} \\
x_{4} & x_{3} & x_{s}
\end{array}\right)_{b}\left(\begin{array}{c|cc}
\alpha_{s} \\
x_{s}
\end{array} \mid \begin{array}{lll}
\alpha_{2} & \alpha_{1} \\
x_{2} & x_{1}
\end{array}\right)_{b}, \\
& \mathcal{E}_{\alpha_{t}}^{(t)}(A \mid X)=\int d x_{t}\left(\begin{array}{c|cc}
\alpha_{4} \\
x_{4}
\end{array} \mid \begin{array}{lll}
\alpha_{t} & \alpha_{1} \\
x_{t} & x_{1}
\end{array}\right)_{b}\left(\begin{array}{c|cc}
\alpha_{t} \\
x_{t}
\end{array} \mid \begin{array}{lll}
\alpha_{3} & \alpha_{2} \\
x_{3} & x_{2}
\end{array}\right)_{b} .
\end{aligned}
$$

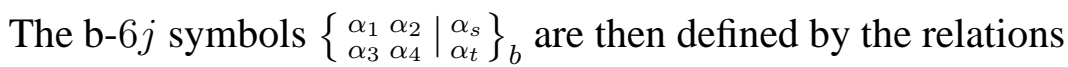

$$
\mathcal{E}_{\alpha_{s}}^{(s)}(A \mid Z)=\int_{Q / 2+\mathrm{iR}} d \mu\left(\alpha_{t}\right)\left\{\begin{array}{ccc}
\alpha_{1} & \alpha_{2} \\
\alpha_{3} \alpha_{4} & \alpha_{\alpha_{t}}
\end{array}\right\}_{b} \mathcal{E}_{\alpha_{t}}^{(t)}(A \mid Z),
$$

where the Plancherel measure $d \mu(\alpha)$ is explicitly given by the expression

$$
d \mu(\alpha)=d \alpha M(\alpha), \quad M(\alpha):=\left|S_{b}(2 \alpha)\right|^{2} .
$$

It is clear that the explicit expression for the b- $6 j$ symbols depends on the normalization chosen for the Clebsch-Gordan maps. We will denote the $6 j$ symbols corresponding to $\left(\begin{array}{ccc}\alpha_{3} \\ x_{3}\end{array} \mid \begin{array}{cc}\alpha_{2} & \alpha_{1} \\ x_{1}\end{array}\right)_{b}^{\text {an }}$

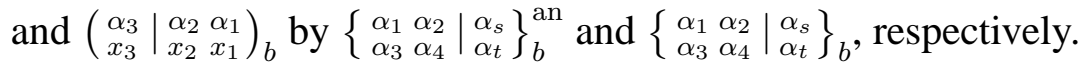

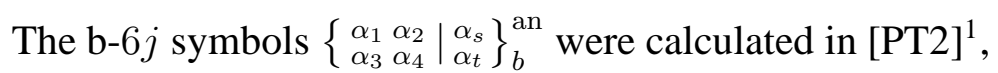

$$
\begin{aligned}
\left\{\begin{array}{c}
\alpha_{1} \\
\alpha_{3} \alpha_{2} \\
\alpha_{3}
\end{array} \mid \begin{array}{l}
\alpha_{s} \\
\alpha_{t}
\end{array}\right\}_{b}^{\text {an }}= & \frac{S_{b}\left(\alpha_{2}+\alpha_{s}-\alpha_{1}\right) S_{b}\left(\alpha_{t}+\alpha_{1}-\alpha_{4}\right)}{S_{b}\left(\alpha_{2}+\alpha_{t}-\alpha_{3}\right) S_{b}\left(\alpha_{s}+\alpha_{3}-\alpha_{4}\right)} \\
& \times \int_{\mathcal{C}} d u S_{b}\left(-\alpha_{2} \pm\left(\alpha_{1}-Q / 2\right)+u\right) S_{b}\left(-\alpha_{4} \pm\left(\alpha_{3}-Q / 2\right)+u\right) \\
& \quad \times S_{b}\left(\alpha_{2}+\alpha_{4} \pm\left(\alpha_{t}-Q / 2\right)-u\right) S_{b}\left(Q \pm\left(\alpha_{s}-Q / 2\right)-u\right) .
\end{aligned}
$$

The following notation has been used $S_{b}(\alpha \pm u):=S_{b}(\alpha+u) S_{b}(\alpha-u)$. The integral in (2.17) will be defined for $\alpha_{k} \in Q / 2+\mathrm{i} \mathbb{R}$ by using a contour $\mathcal{C}$ that approaches $Q+\mathrm{i} \mathbb{R}$ near infinity, and passes the real axis in $(Q / 2, Q)$, and for other values of $\alpha_{k}$ by analytic continuation.

The b- $6 j$ symbols corresponding to the normalization defined above are then given by the formula

$$
\left\{\begin{array}{cc|c}
\alpha_{1} & \alpha_{2} \\
\alpha_{3} & \alpha_{4} & \alpha_{s} \\
\alpha_{t}
\end{array}\right\}_{b}=\frac{M\left(\alpha_{s}, \alpha_{2}, \alpha_{1}\right) M\left(\alpha_{4}, \alpha_{3}, \alpha_{s}\right)}{M\left(\alpha_{t}, \alpha_{3}, \alpha_{2}\right) M\left(\alpha_{4}, \alpha_{t}, \alpha_{1}\right)}\left\{\begin{array}{cc|cc}
\alpha_{1} & \alpha_{2} \\
\alpha_{3} & \alpha_{4} & \alpha_{s} \\
\alpha_{t}
\end{array}\right\}_{b}^{\text {an }} .
$$

with $M\left(\alpha_{3}, \alpha_{2}, \alpha_{1}\right)$ being defined in (2.9).

\footnotetext{
${ }^{1}$ The formula below coincides with equation (228) in [T01] after shifting $s \rightarrow u-\alpha_{s}-Q / 2$. We have moved a factor $\left|S_{b}\left(2 \alpha_{t}\right)\right|^{2}$ into the measure of integration in $(2.15)$.
} 


\section{$2.43 j$ symbols for the modular double}

$3 j$ coefficients describe invariants in tensor products of three representations. Such invariants may be constructed from the Clebsch-Gordon maps and the invariant bilinear form $\mathcal{B}: \mathcal{P}_{\alpha} \otimes$ $\mathcal{P}_{Q-\alpha} \rightarrow \mathbb{C}$ defined by [PT2]

$$
\mathcal{B}(f, g):=\int_{\mathbb{R}} d x f(x) g(x-\mathrm{i} Q / 2) .
$$

We may thereby construct an invariant trilinear form $\mathcal{C}_{\alpha_{3}, \alpha_{2}, \alpha_{1}}: \mathcal{P}_{\alpha_{3}} \otimes \mathcal{P}_{\alpha_{2}} \otimes \mathcal{P}_{\alpha_{1}} \rightarrow \mathbb{C}$ as

$$
\mathcal{C}_{\alpha_{3}, \alpha_{2}, \alpha_{1}}\left(f_{3}, f_{2}, f_{1}\right):=\mathcal{B}\left(f_{3}, \mathrm{C}_{\alpha_{2}, \alpha_{1}}^{Q-\alpha_{3}} \cdot f_{2} \otimes f_{1}\right)
$$

The form $\mathcal{C}_{\alpha_{3}, \alpha_{2}, \alpha_{1}}$ can be represented as

$$
\mathcal{C}_{\alpha_{3}, \alpha_{2}, \alpha_{1}}\left(f_{3}, f_{2}, f_{1}\right)=\int_{\mathbb{R}^{3}} d x_{3} d x_{2} d x_{1}\left(\begin{array}{ccc}
\alpha_{3} & \alpha_{2} & \alpha_{1} \\
x_{3} & x_{2} & x_{1}
\end{array}\right) f_{3}\left(x_{3}\right) f_{2}\left(x_{2}\right) f_{1}\left(x_{1}\right)
$$

with $3 j$-symbols $\left(\begin{array}{lll}\alpha_{3} & \alpha_{2} & \alpha_{1} \\ x_{3} & x_{2} & x_{1}\end{array}\right)$ given in terms of the Clebsch-Gordan coefficients $\left(\begin{array}{l|ll}\alpha_{3} \\ x_{3}\end{array} \mid \begin{array}{ll}\alpha_{2} & \alpha_{1} \\ x_{2} & x_{1}\end{array}\right)$ as

$$
\left(\begin{array}{lll}
\alpha_{3} & \alpha_{2} & \alpha_{1} \\
x_{3} & x_{2} & x_{1}
\end{array}\right)=\left(\begin{array}{l}
Q-\alpha_{3} \\
x_{3}-\mathrm{i} Q / 2
\end{array} \mid \begin{array}{cc}
\alpha_{2} & \alpha_{1} \\
x_{2} & x_{1}
\end{array}\right)
$$

We may similarly define

$$
\begin{aligned}
& { }^{s} \mathcal{C}_{\alpha_{4}, \alpha_{3}, \alpha_{2}, \alpha_{1}}^{\alpha_{s}}\left(f_{4}, f_{3}, f_{2}, f_{1}\right):=\mathcal{B}\left(f f_{4},{ }^{s} C_{\alpha_{3}, \alpha_{2}, \alpha_{1}}^{Q-\alpha_{4}}\left(\alpha_{s}\right) \cdot f_{3} \otimes f_{2} \otimes f_{1}\right), \\
& { }^{t} \mathcal{C}_{\alpha_{4}, \alpha_{3}, \alpha_{2}, \alpha_{1}}^{\alpha_{t}}\left(f_{4}, f_{3}, f_{2}, f_{1}\right):=\mathcal{B}\left(f_{4},{ }^{t} C_{\alpha_{3}, \alpha_{2}, \alpha_{1}}^{Q-\alpha_{4}}\left(\alpha_{t}\right) \cdot f_{3} \otimes f_{2} \otimes f_{1}\right) .
\end{aligned}
$$

The b- $6 j$ symbols $\left\{\begin{array}{lll}\alpha_{1} & \alpha_{2} & \alpha_{s} \\ \alpha_{3} & \alpha_{4} & \alpha_{t}\end{array}\right\}$ are then defined by the relation

$$
{ }^{s} \mathcal{C}_{\alpha_{4}, \alpha_{3}, \alpha_{2}, \alpha_{1}}^{\alpha_{s}}=\int_{Q / 2+\mathrm{i} \mathbb{R}} d \mu\left(\alpha_{t}\right)\left\{\begin{array}{ccc}
\alpha_{1} & \alpha_{2} & \alpha_{s} \\
\alpha_{3} & \alpha_{4} & \alpha_{t}
\end{array}\right\}_{b}{ }^{t} \mathcal{C}_{\alpha_{4}, \alpha_{3}, \alpha_{2}, \alpha_{1}}^{\alpha_{t}}
$$

It follows that

$$
\left\{\begin{array}{lll}
\alpha_{1} & \alpha_{2} & \alpha_{s} \\
\alpha_{3} & \alpha_{4} & \alpha_{t}
\end{array}\right\}_{b}=\left\{\begin{array}{cc|c}
\alpha_{1} & \alpha_{2} & \alpha_{s} \\
\alpha_{3} & \bar{\alpha}_{4} & \alpha_{t}
\end{array}\right\}_{b}, \quad \bar{\alpha}_{4}:=Q-\alpha_{4}
$$

The b-6 $j$ symbols satisfy the following identities [PT1]

$$
\begin{aligned}
& \int_{Q / 2+i \mathbb{R}^{+}} d \mu\left(\delta_{1}\right)\left\{\begin{array}{lll}
\alpha_{1} & \alpha_{2} & \beta_{1} \\
\alpha_{3} & \beta_{2} & \delta_{1}
\end{array}\right\}_{b}\left\{\begin{array}{lll}
\alpha_{1} & \delta_{1} & \beta_{2} \\
\alpha_{4} & \alpha_{5} & \gamma_{2}
\end{array}\right\}_{b}\left\{\begin{array}{lll}
\alpha_{2} & \alpha_{3} & \delta_{1} \\
\alpha_{4} & \gamma_{2} & \gamma_{1}
\end{array}\right\}_{b}=\left\{\begin{array}{lll}
\beta_{1} & \alpha_{3} & \beta_{2} \\
\alpha_{4} & \alpha_{5} & \gamma_{1}
\end{array}\right\}_{b}\left\{\begin{array}{lll}
\alpha_{1} & \alpha_{2} & \beta_{1} \\
\gamma_{1} & \alpha_{5} & \gamma_{2}
\end{array}\right\}_{b}, \\
& \int_{Q / 2+\mathbb{R}^{+}} d \mu\left(\alpha_{s}\right)\left\{\begin{array}{lll}
\alpha_{1} & \alpha_{2} & \alpha_{s} \\
\alpha_{3} & \alpha_{4} & \alpha_{t}
\end{array}\right\}_{b}^{*}\left\{\begin{array}{lll}
\alpha_{1} & \alpha_{2} & \alpha_{s} \\
\alpha_{3} & \alpha_{4} & \alpha_{t}^{\prime}
\end{array}\right\}_{b}=\left(M\left(\alpha_{t}\right)\right)^{-1} \delta\left(\alpha_{t}-\alpha_{t}^{\prime}\right) .
\end{aligned}
$$

The explicit expression will again depend on the chosen normalization of the Clebsch-Gordan maps, giving us two versions, $\left\{\begin{array}{cccc}\alpha_{1} & \alpha_{2} & \alpha_{s} \\ \alpha_{3} & \alpha_{4} & \alpha_{t}\end{array}\right\}_{b}$ and $\left\{\begin{array}{ccc}\alpha_{1} & \alpha_{2} & \alpha_{s} \\ \alpha_{3} & \alpha_{4} & \alpha_{t}\end{array}\right\}_{b}^{\text {an }}$, respectively. 


\subsection{A new integral formula for the $b-6 j$ symbols}

One of our main results will be the following formula for the b- $6 j$ symbols:

$$
\begin{aligned}
& \left\{\begin{array}{rrr}
\alpha_{1} & \alpha_{2} & \alpha_{s} \\
\alpha_{3} & \alpha_{4} & \alpha_{t}
\end{array}\right\}_{b}=\Delta\left(\alpha_{s}, \alpha_{2}, \alpha_{1}\right) \Delta\left(\alpha_{4}, \alpha_{3}, \alpha_{s}\right) \Delta\left(\alpha_{t}, \alpha_{3}, \alpha_{2}\right) \Delta\left(\alpha_{4}, \alpha_{t}, \alpha_{1}\right) \\
& \quad \times \int_{\mathcal{C}} d u S_{b}\left(u-\alpha_{12 s}\right) S_{b}\left(u-\alpha_{s 34}\right) S_{b}\left(u-\alpha_{23 t}\right) S_{b}\left(u-\alpha_{1 t 4}\right) \\
& \quad \times S_{b}\left(\alpha_{1234}-u\right) S_{b}\left(\alpha_{s t 13}-u\right) S_{b}\left(\alpha_{s t 24}-u\right) S_{b}(2 Q-u) .
\end{aligned}
$$

The expression involves the following ingredients:

- We have used the notations $\alpha_{i j k}=\alpha_{i}+\alpha_{j}+\alpha_{k}, \alpha_{i j k l}=\alpha_{i}+\alpha_{j}+\alpha_{k}+\alpha_{l}$.

- $\Delta\left(\alpha_{3}, \alpha_{2}, \alpha_{1}\right)$ is defined as

$$
\Delta\left(\alpha_{3}, \alpha_{2}, \alpha_{1}\right)=\left(\frac{S_{b}\left(\alpha_{1}+\alpha_{2}+\alpha_{s}-Q\right)}{S_{b}\left(\alpha_{1}+\alpha_{2}-\alpha_{s}\right) S_{b}\left(\alpha_{1}+\alpha_{s}-\alpha_{2}\right) S_{b}\left(\alpha_{2}+\alpha_{s}-\alpha_{1}\right)}\right)^{\frac{1}{2}} .
$$

- The integral is defined in the cases that $\alpha_{k} \in Q / 2+\mathrm{i} \mathbb{R}$ by a contour $\mathcal{C}$ which approaches $2 Q+\mathrm{i} \mathbb{R}$ near infinity, and passes the real axis in the interval $(3 Q / 2,2 Q)$. For other values of the variables $\alpha_{k}$ it is defined by analytic continuation.

The reader may notice how closely the structure of the expression in (2.27) resembles the wellknown formulae for the classical $6 j$ symbols.

For establishing this relation, the main step is contained in the following integral identity:

$$
\begin{aligned}
& \left\{\begin{array}{cc|c}
\alpha_{1} & \alpha_{2} & \alpha_{s} \\
\alpha_{3} & \bar{\alpha}_{4} & \alpha_{t}
\end{array}\right\}_{b}^{\text {an }}=\mathcal{C}(\underline{\alpha}) \int_{\mathcal{C}^{\prime}} d u S_{b}\left(u-\alpha_{12 s}\right) S_{b}\left(u-\alpha_{s 34}\right) S_{b}\left(u-\alpha_{23 t}\right) S_{b}\left(u-\alpha_{1 t 4}\right) \\
& \times S_{b}\left(\alpha_{1234}-u\right) S_{b}\left(\alpha_{s t 13}-u\right) S_{b}\left(\alpha_{s t 24}-u\right) S_{b}(2 Q-u),
\end{aligned}
$$

where the contour $\mathcal{C}^{\prime}$ in (2.28) runs between $2 Q-\mathrm{i} \infty$ and $2 Q+\mathrm{i} \infty$, and $\underline{\alpha}$ is shorthand notation for the tuple $\left(\alpha_{1}, \alpha_{2}, \alpha_{3}, \alpha_{4}, \alpha_{s}, \alpha_{t}\right)$. The prefactor $\mathcal{C}(\underline{\alpha})$ is explicitly given by the expression

$$
\begin{aligned}
\mathcal{C}(\underline{\alpha}) & =S_{b}\left(-Q+\alpha_{1}+\alpha_{4}+\alpha_{t}\right) S_{b}\left(Q-\alpha_{1}-\alpha_{2}+\alpha_{s}\right) \\
& \times S_{b}\left(-Q+\alpha_{2}+\alpha_{3}+\alpha_{t}\right) S_{b}\left(Q-\alpha_{2}+\alpha_{3}-\alpha_{t}\right) S_{b}\left(Q+\alpha_{2}-\alpha_{3}-\alpha_{t}\right) \\
& \times S_{b}\left(Q-\alpha_{3}+\alpha_{4}-\alpha_{s}\right) S_{b}\left(Q-\alpha_{3}-\alpha_{4}+\alpha_{s}\right) S_{b}\left(Q+\alpha_{3}-\alpha_{4}-\alpha_{s}\right) .
\end{aligned}
$$

The proof of identity (2.28) is nontrivial. It is described in Appendix B based on recent advances in the theory of elliptic generalizations of the hypergeometric functions [S01, S03, S08]. 


\section{Relations to three-dimensional hyperbolic geometry}

Our goal in this section is to demonstrate by direct calculation that the b- $6 j$ symbols reproduce the volume of non-ideal tetrahedra in the classical limit. A second, perhaps more conceptual proof of this fact will be outlined in section 5 below.

Similar observations concerning relations between the semiclassical behavior of the noncompact quantum dilogarithm and hyperbolic volumes have previously been made in [ $\mathrm{Hi1}, \mathrm{Hi} 2, \mathrm{Hi} 3$, BMS1, BMS2, DGLZ, AK]. It would be interesting to understand the precise relations to our result below.

\subsection{Volumes of non-ideal tetrahedra}

We are considering non-ideal tetrahedra which are completely defined by the collection of six dihedral angles $\eta_{1}, \ldots, \eta_{6}$. In order to formulate the formula for their volumes from [MY], let us use the notation $A_{k}=e^{\mathrm{i} \eta_{k}}$, and define

$$
\begin{aligned}
U(u, \underline{A})= & \mathrm{Li}_{2}(u)+\operatorname{Li}_{2}\left(A_{s t 13} u\right)+\operatorname{Li}_{2}\left(A_{s t 24} u\right)+\operatorname{Li}_{2}\left(A_{1234} u\right) \\
& -\operatorname{Li}_{2}\left(-A_{12 s} u\right)-\mathrm{Li}_{2}\left(-A_{s 34} u\right)-\mathrm{Li}_{2}\left(-A_{4 t 1} u\right)-\mathrm{Li}_{2}\left(-A_{32 t} u\right),
\end{aligned}
$$

where $A_{i j k}:=A_{i} A_{j} A_{k}, A_{i j k l}:=A_{i} A_{j} A_{k} A_{l}$, along with

$$
\begin{aligned}
\Delta(\underline{A})= & \log A_{s} A_{t}+\log A_{2} A_{4}+\log A_{1} A_{3} \\
& +\tilde{\Delta}\left(A_{s}, A_{1}, A_{2}\right)+\tilde{\Delta}\left(A_{s}, A_{3}, A_{3}\right)+\tilde{\Delta}\left(A_{t}, A_{1}, A_{4}\right)+\tilde{\Delta}\left(A_{t}, A_{2}, A_{3}\right),
\end{aligned}
$$

where

$$
\begin{array}{r}
\tilde{\Delta}\left(A_{1}, A_{2}, A_{3}\right)=-\frac{1}{2}\left(\operatorname{Li}_{2}\left(-A_{1} A_{2} A_{3}^{-1}\right)+\operatorname{Li}_{2}\left(-A_{1} A_{2}^{-1} A_{3}\right)+\operatorname{Li}_{2}\left(-A_{1}^{-1} A_{2} A_{3}\right)\right. \\
\left.+\operatorname{Li}_{2}\left(-A_{1}^{-1} A_{2}^{-1} A_{3}^{-1}\right)+\log ^{2} A_{1}+\log ^{2} A_{2}+\log ^{2} A_{3}\right) .
\end{array}
$$

The following formula was found in [MY] Theorem 2]

$$
\operatorname{Vol}(\underline{A})=\frac{1}{2} \operatorname{Im}\left[U\left(u_{+}, \underline{A}\right)+\Delta(\underline{A})\right]=-\frac{1}{2} \operatorname{Im}\left[U\left(u_{-}, \underline{A}\right)+\Delta(\underline{A})\right]
$$

where $u_{ \pm}$are the two roots of the equation

$$
\frac{d U(u, \underline{A})}{d u}=-\frac{2 \pi \mathrm{i}}{u}
$$

It can be shown [MY] that equation (3.4) is a quadratic equation which has two solutions $u_{ \pm}$ which are pure phase, $\left|u_{ \pm}\right|=1$. 


\subsection{Semiclassical limit}

In the following we will assume that $\alpha_{k} \in \mathbb{R}, 0<\alpha_{k}<Q / 2$. In order to study the quasiclassical limit of (2.27) let us write the right hand side of (2.27) in the form

$$
I:=E(\underline{\alpha}) \int_{\mathcal{C}} d u \mathcal{I}(a, b ; u) \text {. }
$$

The integrand $\mathcal{I}(a, b ; u)$ may be written as

$$
\mathcal{I}(a, b ; u)=\frac{\prod_{i=1}^{4} S_{b}\left(a_{i}+u\right)}{S_{b}(-Q+u) \prod_{i=1}^{3} S_{b}\left(Q-b_{i}+u\right)} d u,
$$

where

$$
\begin{aligned}
& a \equiv\left[a_{1}, a_{2}, a_{3}, a_{4}\right]=\left[-\alpha_{s}-\alpha_{1}-\alpha_{2},-\alpha_{s}-\alpha_{3}-\alpha_{4},-\alpha_{t}-\alpha_{1}-\alpha_{4},-\alpha_{t}-\alpha_{2}-\alpha_{3}\right], \\
& b \equiv\left[b_{1}, b_{2}, b_{3}\right]=\left[\alpha_{s}+\alpha_{t}+\alpha_{1}+\alpha_{3}, \alpha_{s}+\alpha_{t}+\alpha_{2}+\alpha_{4}, \alpha_{1}+\alpha_{2}+\alpha_{3}+\alpha_{4}\right] .
\end{aligned}
$$

The quasi-classical limit of $\mathcal{I}(a, b ; u)$ is easily determined with the help of formula (A.17) in Appendix A. In order to write the result in an convenient form let us reparameterize variables

$$
e^{-2 \pi \mathrm{i} b \alpha_{k}+\pi \mathrm{i}} \equiv A_{k}, \quad k \in\{1,2,3,4, s, t\} .
$$

Introducing the integration variables $v:=2 \pi b(u-Q / 2)$ we get an integral of the form

$$
I=D(\underline{\alpha}) \int_{\mathcal{C}-Q / 2} \frac{d v}{2 \pi b} \mathcal{J}(a, b ; v)
$$

whose integrand $\mathcal{J}(a, b ; v)$ has quasi-classical asymptotics

$$
\mathcal{J}(a, b ; v)=\exp \left(\frac{1}{2 \pi b^{2}} U\left(e^{\mathrm{i} v}, \underline{A}\right)\right)\left(1+\mathcal{O}\left(b^{2}\right)\right)
$$

with $U\left(e^{\mathrm{i} v}, \underline{A}\right)$ given by the formula $(\underline{3.1})$. The quasiclassical asymptotics of the prefactor in (3.8) is

$$
D_{\mathrm{cl}}(\underline{A})=\exp \left(\frac{1}{2 \pi \mathrm{i} b^{2}}\left(\Delta(\underline{A})-\frac{5}{3} \pi^{2}\right)\right),
$$

where $\Delta(\underline{A})$ was defined in $(\underline{3.2})$ above.

Now we are ready to perform the saddle-point approximation for the integral (3.5). The saddle points are the solutions of the equation (3.4). The values of the $b-6 j$ at these points are

$$
\exp \left(\frac{1}{2 \pi \mathrm{i} b^{2}} W_{ \pm}(\underline{A})\right), \quad \text { where } \quad W_{ \pm}(\underline{A})=U\left(z_{ \pm}, \underline{A}\right)+\Delta(\underline{A})-\frac{5}{3} \pi^{2}+2 \pi \mathrm{i} \log u_{ \pm} .
$$

Since $u_{ \pm}=e^{ \pm \pi \mathrm{i} \phi}, \phi \in \mathbb{R}$ as noted above, we find that

$$
W_{ \pm}(\underline{A})=U\left(z_{ \pm}, \underline{A}\right)+\Delta(\underline{A})-\frac{5}{3} \pi^{2} \mp 2 \pi^{2} \phi .
$$

Taking the imaginary part of (3.11) one sees that we are getting the volume of a hyperbolic tetrahedron (3.3). 


\section{Relation to Liouville theory and the representation theory of $\operatorname{Diff}\left(\mathrm{S}^{1}\right)$}

In this section we want to explain that the normalization leading to the definition of the $b-6 j$ symbols is also very natural from the point of view of Liouville theory. This is closely related to the interpretation of b- $6 j$ symbols as $6 j$ symbols for the the infinite-dimensional group Diff $\left(\mathrm{S}^{1}\right)$.

\subsection{Fusion kernel}

Recall that the fusion kernel is usually defined in terms of the conformal blocks appearing in the holomorphically factorized form of the four-point functions,

$$
\begin{aligned}
& \left\langle V_{\alpha_{4}}\left(z_{4}, \bar{z}_{4}\right) V_{\alpha_{3}}\left(z_{3}, \bar{z}_{3}\right) V_{\alpha_{2}}\left(z_{2}, \bar{z}_{2}\right) V_{\alpha_{1}}\left(z_{1}, \bar{z}_{1}\right)\right\rangle= \\
& \quad=\int_{Q / 2+\mathrm{i} \mathbb{R}} d \alpha_{s} C\left(\alpha_{4}, \alpha_{3}, \alpha_{s}\right) C\left(Q-\alpha_{s}, \alpha_{2}, \alpha_{1}\right) \mathcal{F}_{\alpha_{s}}^{(s)}(A \mid Z) \mathcal{F}_{\alpha_{s}}^{(s)}(A \mid \bar{Z}) \\
& \quad=\int_{Q / 2+\mathrm{i} \mathbb{R}} d \alpha_{t} C\left(\alpha_{4}, \alpha_{t}, \alpha_{1}\right) C\left(Q-\alpha_{t}, \alpha_{3}, \alpha_{2}\right) \mathcal{F}_{\alpha_{t}}^{(t)}(A \mid Z) \mathcal{F}_{\alpha_{t}}^{(t)}(A \mid \bar{Z})
\end{aligned}
$$

where $A=\left(\alpha_{1}, \alpha_{2}, \alpha_{3}, \alpha_{4}\right), Z=\left(z_{1}, z_{2}, z_{3}, z_{4}\right)$, and

$$
\begin{aligned}
C\left(\alpha_{1}, \alpha_{2}, \alpha_{3}\right)=\left(\pi \mu \gamma\left(b^{2}\right) b^{2-2 b^{2}}\right)^{\frac{1}{b}\left(Q-\alpha_{1}-\alpha_{2}-\alpha_{3}\right)} \times \\
\quad \times \frac{\Upsilon_{0} \Upsilon\left(2 \alpha_{1}\right) \Upsilon\left(2 \alpha_{2}\right) \Upsilon\left(2 \alpha_{3}\right)}{\Upsilon\left(\alpha_{1}+\alpha_{2}+\alpha_{3}-Q\right) \Upsilon\left(\alpha_{1}+\alpha_{3}-\alpha_{2}\right) \Upsilon\left(\alpha_{1}+\alpha_{2}-\alpha_{3}\right) \Upsilon\left(\alpha_{2}+\alpha_{3}-\alpha_{1}\right)},
\end{aligned}
$$

here $\mu$ is the so-called cosmological constant in Liouville field theory and $\gamma(x)=\Gamma(x) / \Gamma(1-$ $x)$. We also used $\Upsilon(x)=\left(\Gamma_{b}(x) \Gamma_{b}(Q-x)\right)^{-1}, \Upsilon_{0}=\left.\frac{d \Upsilon(x)}{d x}\right|_{x=0}$ where the function $\Gamma_{b}(x)$ is the Barnes double Gamma function. Appendix A lists the definition and the relevant properties of $\Gamma_{b}(x)$.

The first expression (4.1) for the four-point functions represents the operator product expansion of the fields $V_{\alpha_{2}}\left(z_{2}, \bar{z}_{2}\right)$ and $V_{\alpha_{1}}\left(z_{1}, \bar{z}_{1}\right)$, while the second expression (4.2) represents the operator product expansion of the fields $V_{\alpha_{3}}\left(z_{3}, \bar{z}_{3}\right)$ and $V_{\alpha_{2}}\left(z_{2}, \bar{z}_{2}\right)$. The equality of the two expressions (4.1) and (4.2) follows from the validity of the relations

$$
\mathcal{F}_{\alpha_{s}}^{(s)}(A \mid Z)=\int_{Q / 2+\mathrm{i} \mathbb{R}} d \alpha_{t} F_{\alpha_{s} \alpha_{t}}\left[\begin{array}{cc}
\alpha_{3} & \alpha_{2} \\
\alpha_{4} & \alpha_{1}
\end{array}\right] \mathcal{F}_{\alpha_{t}}^{(t)}(A \mid Z),
$$

which were established in [T01]. The following formula was found in [PT1, T01],

$$
F_{\alpha_{s} \alpha_{t}}\left[\begin{array}{cc}
\alpha_{3} & \alpha_{2} \\
\alpha_{4} & \alpha_{1}
\end{array}\right]=\frac{N\left(\alpha_{s}, \alpha_{2}, \alpha_{1}\right) N\left(\alpha_{4}, \alpha_{3}, \alpha_{s}\right)}{N\left(\alpha_{t}, \alpha_{3}, \alpha_{2}\right) N\left(\alpha_{4}, \alpha_{t}, \alpha_{1}\right)} M\left(\alpha_{t}\right)\left\{\begin{array}{cc|c}
\alpha_{1} & \alpha_{2} & \alpha_{s} \\
\alpha_{3} & \alpha_{4} & \alpha_{t}
\end{array}\right\}^{\text {an }},
$$

where

$$
\begin{aligned}
& N\left(\alpha_{3}, \alpha_{2}, \alpha_{1}\right)= \\
& =\frac{\Gamma_{b}\left(2 Q-2 \alpha_{3}\right) \Gamma_{b}\left(2 \alpha_{2}\right) \Gamma_{b}\left(2 \alpha_{1}\right)}{\Gamma_{b}\left(2 Q-\alpha_{1}-\alpha_{2}-\alpha_{3}\right) \Gamma_{b}\left(Q-\alpha_{1}-\alpha_{2}+\alpha_{3}\right) \Gamma_{b}\left(\alpha_{1}+\alpha_{3}-\alpha_{2}\right) \Gamma_{b}\left(\alpha_{2}+\alpha_{3}-\alpha_{1}\right)}
\end{aligned}
$$




\subsection{Unitary normalization}

The expressions (4.1) and (4.2) strongly suggest to redefine the conformal blocks by absorbing the three-point functions $C\left(\alpha_{3}, \alpha_{2}, \alpha_{1}\right)$ into the definition,

$$
\begin{gathered}
\mathcal{G}_{\alpha_{s}}^{(s)}(A \mid Z):=\left(C\left(\alpha_{4}, \alpha_{3}, \alpha_{s}\right) C\left(Q-\alpha_{s}, \alpha_{2}, \alpha_{1}\right)\right)^{\frac{1}{2}} \mathcal{F}_{\alpha_{s}}^{(s)}(A \mid Z), \\
\mathcal{G}_{\alpha_{t}}^{(t)}(A \mid Z):=\left(C\left(\alpha_{4}, \alpha_{t}, \alpha_{1}\right) C\left(Q-\alpha_{t}, \alpha_{3}, \alpha_{2}\right)\right)^{\frac{1}{2}} \mathcal{F}_{\alpha_{t}}^{(t)}(A \mid Z) .
\end{gathered}
$$

This corresponds to normalizing the conformal blocks associated to the three-punctured sphere in such a way that their scalar product is always unity. This normalization may be called the unitary normalization. We then have

$$
\begin{aligned}
& \left\langle V_{\alpha_{4}}\left(z_{4}, \bar{z}_{4}\right) V_{\alpha_{3}}\left(z_{3}, \bar{z}_{3}\right) V_{\alpha_{2}}\left(z_{2}, \bar{z}_{2}\right) V_{\alpha_{1}}\left(z_{1}, \bar{z}_{1}\right)\right\rangle= \\
& \quad=\int_{\frac{Q}{2}+\mathrm{i} \mathbb{R}} d \alpha_{s} \mathcal{G}_{\alpha_{s}}^{(s)}(A \mid Z) \mathcal{G}_{\alpha_{s}}^{(s)}(A \mid \bar{Z})=\int_{\frac{Q}{2}+\mathrm{i} \mathbb{R}} d \alpha_{t} \mathcal{G}_{\alpha_{t}}^{(t)}(A \mid Z) \mathcal{G}_{\alpha_{t}}^{(t)}(A \mid \bar{Z}),
\end{aligned}
$$

the second equation being a consequence of the unitarity of the change of basis

$$
\mathcal{G}_{\alpha_{s}}^{(s)}(A \mid Z)=\int_{Q / 2+\mathrm{i} \mathbb{R}} d \alpha_{t} G_{\alpha_{s} \alpha_{t}}\left[\begin{array}{cc}
\alpha_{3} & \alpha_{2} \\
\alpha_{4} & \alpha_{1}
\end{array}\right] \mathcal{G}_{\alpha_{t}}^{(t)}(A \mid Z)
$$

The normalized fusion coefficients $G_{\alpha_{s} \alpha_{t}}\left[\begin{array}{cc}\alpha_{3} & \alpha_{2} \\ \alpha_{4} & \alpha_{1}\end{array}\right]$ are related to the $F_{\alpha_{s} \alpha_{t}}\left[\begin{array}{ll}\alpha_{3} & \alpha_{2} \\ \alpha_{4} & \alpha_{1}\end{array}\right]$ as

$$
G_{\alpha_{s} \alpha_{t}}\left[\begin{array}{cc}
\alpha_{3} & \alpha_{2} \\
\alpha_{4} & \alpha_{1}
\end{array}\right]=\sqrt{\frac{C\left(\alpha_{4}, \alpha_{3}, \alpha_{s}\right) C\left(Q-\alpha_{s}, \alpha_{2}, \alpha_{1}\right)}{C\left(\alpha_{4}, \alpha_{t}, \alpha_{1}\right) C\left(Q-\alpha_{t}, \alpha_{3}, \alpha_{2}\right)}} F_{\alpha_{s} \alpha_{t}}\left[\begin{array}{cc}
\alpha_{3} & \alpha_{2} \\
\alpha_{4} & \alpha_{1}
\end{array}\right] .
$$

The fusion coefficients $G_{\alpha_{s} \alpha_{t}}\left[\begin{array}{ll}\alpha_{3} & \alpha_{2} \\ \alpha_{4} & \alpha_{1}\end{array}\right]$ have a simple expression in terms of the b- $6 j$ symbols,

$$
G_{\alpha_{s} \alpha_{t}}\left[\begin{array}{ccc}
\alpha_{3} & \alpha_{2} \\
\alpha_{4} & \alpha_{1}
\end{array}\right]=\left(M\left(\alpha_{t}\right) M\left(\alpha_{s}\right)\right)^{\frac{1}{2}}\left\{\begin{array}{ccc}
\alpha_{1} & \alpha_{2} & \alpha_{s} \\
\alpha_{3} & \alpha_{4} & \alpha_{t}
\end{array}\right\}_{b} .
$$

Indeed, formula (4.11) is a straightforward consequence of equations (4.10), (4.5) and (2.18) above.

\subsection{6j symbols of $\operatorname{Diff}\left(S^{1}\right)$}

It is known that Liouville theory is deeply related to the representation theory of the group $\operatorname{Diff}\left(S^{1}\right)$ of diffeomorphisms of the unit circle [T08]. The operator product expansion from conformal field theory leads to the definition of a suitable generalization of the tensor product operation for representations of infinite-dimensional groups like $\operatorname{Diff}\left(S^{1}\right)$. One may therefore interpret the chiral vertex-operators from conformal field theory as analogs of the ClebschGordan maps, and the fusion coefficients as analog of $6 j$-symbols [MS, T01, T08].

A similar issue arises here as pointed out above in our discussion of the modular double: To find particularly natural normalization conditions. The normalization defined in (4.7) above, 
while being natural from the physical point of view, is not a direct counterpart of the normalization condition used to define the $6 j$ symbols of the modular double above. Such a normalization condition can naturally be defined by requiring invariance under the Weyl-reflections $\alpha_{i} \rightarrow Q-\alpha_{i}$. Due to the factors $\Upsilon\left(2 \alpha_{i}\right)$ in the definition of $C\left(\alpha_{3}, \alpha_{2}, \alpha_{1}\right)$, the symmetry under $\alpha_{i} \rightarrow Q-\alpha_{i}$ is spoiled by the change of normalization (4.7).

However, it is easy to restore this symmetry by replacing the normalization factor $C\left(\alpha_{3}, \alpha_{2}, \alpha_{1}\right)$ entering the definition (4.7) by

$$
D\left(\alpha_{1}, \alpha_{2}, \alpha_{3}\right)=\frac{\left|\Gamma_{b}\left(2 \alpha_{1}\right) \Gamma_{b}\left(2 \alpha_{2}\right) \Gamma_{b}\left(2 \alpha_{3}\right)\right|^{-2}}{\Upsilon\left(2 \alpha_{1}\right) \Upsilon\left(2 \alpha_{2}\right) \Upsilon\left(2 \alpha_{3}\right)} C\left(\alpha_{3}, \alpha_{2}, \alpha_{1}\right)
$$

Replacing $C$ by $D$ in (4.7) leads to the definition of normalized conformal blocks $\mathcal{K}_{\alpha_{s}}^{(s)}(A \mid Z)$ and $\mathcal{K}_{\alpha_{t}}^{(t)}(A \mid Z)$ which can be interpreted as analogs of invariants in tensor products of four representations of $\operatorname{Diff}\left(S^{1}\right)$. The kernel appearing in the relation

$$
\mathcal{K}_{\alpha_{s}}^{(s)}(A \mid Z)=\int_{Q / 2+\mathrm{i} \mathbb{R}} d \mu\left(\alpha_{t}\right)\left\{\begin{array}{ccc}
\alpha_{1} & \alpha_{2} & \alpha_{s} \\
\alpha_{3} & \alpha_{4} & \alpha_{t}
\end{array}\right\}_{\operatorname{Diff}\left(\mathrm{S}^{1}\right)} \mathcal{K}_{\alpha_{t}}^{(t)}(A \mid Z)
$$

is naturally interpreted as an analog of the $6 j$ symbols for $\operatorname{Diff}\left(S^{1}\right)$. It coincides exactly with the b- $6 j$ symbols,

$$
\left\{\begin{array}{lll}
\alpha_{1} & \alpha_{2} & \alpha_{s} \\
\alpha_{3} & \alpha_{4} & \alpha_{t}
\end{array}\right\}_{\operatorname{Diff}\left(\mathrm{S}^{1}\right)}=\left\{\begin{array}{lll}
\alpha_{1} & \alpha_{2} & \alpha_{s} \\
\alpha_{3} & \alpha_{4} & \alpha_{t}
\end{array}\right\}_{b}
$$

as can easily be checked by straightforward calculations.

\section{Application to two-dimensional quantum hyperbolic geometry}

It is known that the Racah-Wigner symbols of the modular double play an important role when the quantum Teichmüller theory [Fo97, Ka98, CF99] is studied in the length representation [T03, T05]. Having fixed a particular normalization in our definition of the b- $6 j$ symbols above naturally leads to question what it corresponds to in this context. We are going to show that the definition of the b- $6 j$ symbols corresponds to the quantization of a particular choice of Darbouxcoordinates for the classical Teichmüller spaces. The Teichmüller spaces $\mathcal{T}(C)$ are well-known to be related to a connected component in the moduli space of flat $S L(2, \mathbb{R})$-connections on Riemann surfaces. Natural Darboux coordinates for this space have recently been discussed in [NRS].

The quantization of the Teichmüller spaces will be discussed in terms of the Darboux coordinates of $[\mathrm{NRS}]$ in a self-contained manner in [TeVa] . In the following we will collect some relevant observations that can fairly easily be extracted from the existing literature. 


\subsection{Classical Teichmüller theory of the four-holed sphere}

To be specific, let us restrict attention to four-holed spheres $C_{0,4}$. The holes are assumed to be represented by geodesics with lengths $L=\left(l_{1}, \ldots, l_{4}\right)$. There are three simple closed curves $\gamma_{s}, \gamma_{t}$, and $\gamma_{u}$ encircling pairs of points $\left(z_{1}, z_{2}\right),\left(z_{2}, z_{3}\right)$ and $\left(z_{1}, z_{3}\right)$, respectively. A set of useful coordinate functions are defined in terms of the hyperbolic cosines $L_{\sigma}=2 \cosh \frac{l_{\sigma}}{2}$, $\sigma \in\{s, t, u\}$, of the geodesic length functions $l_{\sigma}$ on $\mathcal{T}_{0,4} \equiv \mathcal{T}\left(C_{0,4}\right) . l_{\sigma}$ is defined as the length of the geodesic $\gamma_{\sigma}$, defined by means of the constant negative curvature metric on $C_{0,4}$.

The well-known relations between Teichmüller spaces $\mathcal{T}(C)$ and the moduli spaces $\mathcal{M}_{G}(C)$ of flat $G=S L(2, \mathbb{R})$-connections on Riemann surfaces imply that the geodesic length functions $L_{\sigma}$ are related to the holonomies $g_{\sigma}$ along $\gamma_{\sigma}$ as $L_{\sigma}=-\operatorname{Tr}\left(g_{\sigma}\right)$. This allows us to use the description given in [NRS], which may be briefly summarized as follows. The structure of $\mathcal{M}_{G}\left(C_{0,4}\right)$ as an algebraic variety is expressed by the fact that the three coordinate functions $L_{s}, L_{t}$ and $L_{u}$ satisfy one algebraic relation of the form $\mathcal{P}_{L}\left(L_{s}, L_{t}, L_{t}\right)=0$. The Poisson bracket $\left\{L_{\sigma_{1}}, L_{\sigma_{2}}\right\}$ defined by the Weil-Petersson symplectic form is also algebraic in the length variables $L_{\sigma}$, and can be written elegantly in the form

$$
\left\{L_{s}, L_{t}\right\}=\frac{\partial}{\partial L_{u}} \mathcal{P}_{L}\left(L_{s}, L_{t}, L_{t}\right) .
$$

As shown in [NRS] one may represent $L_{s}, L_{t}$ and $L_{u}$ in terms of Darboux-coordinates $l_{s}$ and $k_{s}$ which have Poisson bracket $\left\{l_{s}, k_{s}\right\}=2$. The expressions for $L_{s}$ and $L_{t}$ are, in particular,

$$
\begin{aligned}
& L_{s}=2 \cosh \left(l_{s} / 2\right), \\
& L_{t}\left(L_{s}^{2}-4\right)=2\left(L_{2} L_{3}+L_{1} L_{4}\right)+L_{s}\left(L_{1} L_{3}+L_{2} L_{4}\right)+2 \cosh \left(k_{s}\right) \sqrt{c_{12}\left(L_{s}\right) c_{34}\left(L_{s}\right)},
\end{aligned}
$$

where $L_{i}=2 \cosh \frac{l_{i}}{2}$, and $c_{i j}\left(L_{s}\right)$ is defined as

$$
\begin{aligned}
c_{i j}\left(L_{s}\right) & =L_{s}^{2}+L_{i}^{2}+L_{j}^{2}+L_{s} L_{i} L_{j}-4 \\
& =2 \cosh \frac{l_{s}+l_{i}+l_{j}}{4} 2 \cosh \frac{l_{s}+l_{i}-l_{j}}{4} 2 \cosh \frac{l_{s}-l_{i}+l_{j}}{4} 2 \cosh \frac{l_{s}-l_{i}-l_{j}}{4} .
\end{aligned}
$$

Together with a similar formula for $L_{u}$, these expressions ensure that both the algebraic relation $\mathcal{P}_{L}\left(L_{s}, L_{t}, L_{t}\right)=0$ and the Poisson structure (5.1) are satisfied. These Darboux coordinates are identical to the Fenchel-Nielsen length-twist coordinates well-known in hyperbolic geometry 2

Similar Darboux coordinates $\left(l_{t}, k_{t}\right)$ and $\left(l_{u}, k_{u}\right)$ can be associated to the curves $\gamma_{t}$ and $\gamma_{u}$, respectively. The change of coordinates between the Darboux-coordinates $\left(l_{s}, k_{s}\right)$ and $\left(l_{t}, k_{t}\right)$ is represented by a generating function $\mathcal{S}_{L}^{s t}\left(l_{s}, l_{t}\right)$ such that

$$
\frac{\partial}{\partial l_{s}} \mathcal{S}_{L}^{s t}\left(l_{s}, l_{t}\right)=-k_{s}, \quad \frac{\partial}{\partial l_{t}} \mathcal{S}_{L}^{s t}\left(l_{s}, l_{t}\right)=k_{t} .
$$

\footnotetext{
${ }^{2}$ This can be inferred from [ALPS]. We thank T. Dimofte for pointing this reference out to us
} 
Other natural sets of Darboux-coordinates $\left(l_{\sigma}, k_{\sigma}^{\prime}\right)$ can be obtained by means of canonical transformations $k_{\sigma}^{\prime}=k_{\sigma}+f\left(l_{\sigma}\right)$. By a suitable choice of $f(\sigma)$, one gets Darboux coordinates $\left(l_{s}, k_{s}^{\prime}\right)$ in which the expression for $L_{t}$ in (5.2) is replaced by

$$
\begin{aligned}
L_{t}\left(L_{s}^{2}-4\right)= & 2\left(L_{2} L_{3}+L_{1} L_{4}\right)+L_{s}\left(L_{1} L_{3}+L_{2} L_{4}\right) \\
& +2 \cosh \frac{l_{s}+l_{1}-l_{2}}{4} 2 \cosh \frac{l_{s}+l_{2}-l_{1}}{4} 2 \cosh \frac{l_{s}+l_{3}-l_{4}}{4} 2 \cosh \frac{l_{s}+l_{4}-l_{3}}{4} e^{+k_{s}^{\prime}} \\
& +2 \cosh \frac{l_{s}+l_{1}+l_{2}}{4} 2 \cosh \frac{l_{s}-l_{1}-l_{2}}{4} 2 \cosh \frac{l_{s}+l_{3}+l_{4}}{4} 2 \cosh \frac{l_{s}-l_{3}-l_{4}}{4} e^{-k_{s}^{\prime}} .
\end{aligned}
$$

The Darboux coordinates $\left(l_{s}, k_{s}^{\prime}\right)$ are equally good to represent the Poisson structure of $\mathcal{M}_{G}\left(C_{0,4}\right)$, but they have the advantage that the expressions for $L_{\sigma}$ do not contain square-roots. This will later turn out to be important.

\subsection{The quantization problem}

The quantum Teichmüller theory [Fo97, Ka98, CF99, CF00] constructs a non-commutative algebra $\mathcal{A}_{b}$ deforming the Poisson-algebra of geodesic length functions on Teichmüller space. In the so-called length representation [T03, T05] one may construct natural representations of this algebra associated to pants decompositions of the Riemann surface under consideration.

For the case under consideration, the aim is to construct a one-parameter family of noncommutative deformations $\mathcal{A}_{b}$ of the Poisson-algebra of functions on $\mathcal{T}_{0,4} \equiv \mathcal{T}\left(C_{0,4}\right)$ which has generators $\mathcal{L}_{s}, \mathcal{L}_{t}, \mathcal{L}_{u}$ corresponding to the functions $L_{\sigma}, \sigma \in\{s, t, u\}$, respectively. There is one algebraic relation that should be satisfied among the three generators $\mathcal{L}_{s}, \mathcal{L}_{t}, \mathcal{L}_{u}$.

Natural representations $\pi_{\sigma}, \sigma \in\{s, t, u\}$, of $\mathcal{A}_{b}$ by operators on suitable spaces of functions $\psi_{\sigma}\left(l_{\sigma}\right)$ can be constructed in terms of the quantum counterparts of the Darboux variables $l_{\sigma}, k_{\sigma}$, now represented by the operators $\mathrm{I}_{\sigma}, \mathrm{k}_{\sigma}$ defined as

$$
\mathrm{I}_{\sigma} \psi_{\sigma}\left(l_{\sigma}\right):=l_{\sigma} \psi_{\sigma}\left(l_{\sigma}\right), \quad \mathrm{k}_{s} \psi_{\sigma}\left(l_{\sigma}\right):=4 \pi b^{2} \frac{1}{\mathrm{i}} \frac{\partial}{\partial l_{s}} \psi_{\sigma}\left(l_{\sigma}\right)
$$

The operator $\pi_{\sigma}\left(\mathcal{L}_{\sigma}\right)$ acts as operator of multiplication in the representation $\pi_{\sigma}, \pi_{\sigma}\left(\mathcal{L}_{\sigma}\right) \equiv$ $2 \cosh \left(\mathrm{I}_{\sigma}\right) / 2$. The remaining two generators of $\mathcal{A}_{b}$ are then represented as difference operators. Considering the representation $\pi_{s}$, for example, we will find that $\pi_{s}\left(\mathcal{L}_{t}\right)$ can be represented in the form

$$
\pi_{s}\left(\mathcal{L}_{t}\right) \psi_{s}\left(l_{s}\right)=\left[D_{+}\left(l_{s}\right) e^{+\mathrm{k}_{s}}+D_{0}\left(l_{s}\right)+D_{-}\left(l_{s}\right) e^{-\mathrm{k}_{s}}\right] \psi_{s}\left(l_{s}\right) .
$$

This formula should of course reproduce (5.2) or (5.5) in the classical limit, but due to ordering issues and other possible quantum corrections it is a priori far from obvious how to define the coefficients $D_{\epsilon}\left(l_{s}\right), \epsilon=-, 0,+$. 
Note, in particular, that the requirement that $\pi_{s}\left(\mathcal{L}_{s}\right)$ acts as multiplication operator leaves a large freedom. A gauge transformation

$$
\psi_{s}\left(l_{s}\right)=e^{\mathrm{i} \chi\left(l_{s}\right)} \psi_{s}^{\prime}\left(l_{s}\right)
$$

would lead to a representation $\pi_{s}^{\prime}$ of the form (5.7) with $\mathrm{k}_{s}$ replaced by

$$
\mathrm{k}_{s}^{\prime}:=\mathrm{k}_{s}+4 \pi b^{2} \partial_{l_{s}} \chi\left(l_{s}\right)
$$

This is nothing but the quantum version of a canonical transformation $\left(l_{s}, k_{s}\right) \rightarrow\left(l_{s}, k_{s}+f\left(l_{s}\right)\right)$. The representation $\pi_{s}^{\prime}\left(\mathcal{L}_{t}\right)$ may then be obtained from (5.7) by replacing $D_{\epsilon}\left(l_{s}\right) \rightarrow E_{\epsilon}\left(l_{s}\right)$ with $E_{\epsilon}\left(l_{s}\right)$ equal to $e^{\mathrm{i}\left(\chi\left(l_{s}-4 \epsilon \mathrm{i} \pi b^{2}\right)-\chi\left(l_{s}\right)\right)} D_{\epsilon}\left(l_{s}\right)$ for $\epsilon=-1,0,1$. Fixing a particular set of Darboux coordinates corresponds to fixing a particular choice of the coefficients $D_{\epsilon}\left(l_{s}\right)$ in 5.7).

\subsection{Transitions between representation}

The transition between any pair of representations $\pi_{\sigma_{1}}$ and $\pi_{\sigma_{2}}$ can be represented as an integral transformation of the form

$$
\psi_{\sigma_{1}}\left(l_{\sigma_{1}}\right)=\int d l_{\sigma_{2}} A_{L}^{\sigma_{1} \sigma_{2}}\left(l_{\sigma_{1}}, l_{\sigma_{2}}\right) \psi_{\sigma_{2}}\left(l_{\sigma_{2}}\right)
$$

The relations

$$
\begin{aligned}
& \left(\pi_{s}\left(\mathrm{k}_{t}\right) \psi_{s}\right)\left(l_{s}\right)=4 \pi b^{2} \int d l_{t} A_{L}^{s t}\left(l_{s}, l_{t}\right) \frac{1}{\mathrm{i}} \frac{\partial}{\partial l_{t}} \psi_{t}\left(l_{t}\right), \\
& 4 \pi b^{2} \frac{1}{\mathrm{i}} \frac{\partial}{\partial l_{s}} \psi_{s}\left(l_{s}\right)=\int d l_{t} A_{L}^{s t}\left(l_{s}, l_{t}\right)\left(\pi_{t}\left(\mathrm{k}_{s}\right) \psi_{t}\right)\left(l_{t}\right),
\end{aligned}
$$

describing the quantum change of Darboux coordinates are direct consequences.

It is important to note that the problem to find the proper quantum representation of the generators $\pi_{\sigma}\left(\mathcal{L}_{\sigma^{\prime}}\right)$ is essentially equivalent to the problem to find the kernels $A_{L}^{\sigma_{1} \sigma_{2}}\left(l_{\sigma_{1}}, l_{\sigma_{2}}\right)$ in (5.10). Indeed, the requirement that $\pi_{\sigma}\left(\mathcal{L}_{\sigma}\right) \equiv 2 \cosh \left(\mathrm{I}_{\sigma}\right) / 2$ implies difference equations for the kernel $A_{L}^{\sigma_{1} \sigma_{2}}\left(l_{\sigma_{1}}, l_{\sigma_{2}}\right)$ such as

$$
\pi_{\sigma_{1}}\left(\mathcal{L}_{\sigma_{2}}\right) \cdot A_{L}^{\sigma_{1} \sigma_{2}}\left(l_{\sigma_{1}}, l_{\sigma_{2}}\right)=2 \cosh \left(l_{\sigma_{2}} / 2\right) A_{L}^{\sigma_{1} \sigma_{2}}\left(l_{\sigma_{1}}, l_{\sigma_{2}}\right)
$$

The difference operator on the left is of course understood to act on the variable $l_{\sigma_{1}}$ only. Under certain natural conditions one may show that the difference equations (5.12) determine the kernels $A_{L}^{\sigma_{1} \sigma_{2}}\left(l_{\sigma_{1}}, l_{\sigma_{2}}\right)$ uniquely. Conversely, knowing $A_{L}^{\sigma_{1} \sigma_{2}}\left(l_{\sigma_{1}}, l_{\sigma_{2}}\right)$, one may show [TeVa] that it satisfies relations of the form (5.12), and thereby deduce the explicit form of $\pi_{\sigma_{1}}\left(\mathcal{L}_{\sigma_{2}}\right)$.

Considering the generalization to Riemann spheres $C_{0, n}$ with more than four holes it is natural to demand that the full theory can be built in a uniform manner from the local pieces associated 
to the four-holed spheres that appear in a pants decomposition of $C_{0, n}$. This leads to severe restrictions on the kernels $A_{L}^{s t}\left(l_{s}, l_{t}\right)$ known as the pentagon- and hexagon equations [T05]. We claim that the resulting constraints determine $A_{L}^{s t}\left(l_{s}, l_{t}\right)$ essentially uniquely up to changes of the normalization associated to pairs of pants.

Solutions of these conditions are clearly given by the b- $6 j$-symbols. It is important to note, however, that a change of normalization of the form (2.18) will be equivalent to a gauge transformation (5.8). This means that different normalizations of the b- $6 j$ symbols are in one-to-one correspondence with choices of Darboux-coordinates $\left(l_{\sigma}^{\prime}, k_{\sigma}^{\prime}\right)$ obtained from $\left(l_{\sigma}, k_{\sigma}\right)$ by canonical transformations of the form $l_{\sigma}^{\prime}=l_{\sigma}, k_{\sigma}^{\prime}=k_{\sigma}+f\left(l_{\sigma}\right)$. Only a very particular normalization for the b- $6 j$ symbols can correspond to the quantization of the Fenchel-Nielsen coordinates.

\subsection{Quantization of Fenchel-Nielsen coordinates}

The main observation we want to make here may be summarized in the following two statements:

1) The geodesic length operators can be represented in terms of the quantized Fenchel-Nielsen coordinates as follows:

$$
\begin{aligned}
\pi_{s}^{\operatorname{can}}\left(\mathcal{L}_{s}\right)= & 2 \cosh \left(\mathrm{I}_{s} / 2\right) \\
\pi_{s}^{\operatorname{can}}\left(\mathcal{L}_{t}\right)= & \frac{1}{2\left(\cosh \mathrm{I}_{s}-\cos 2 \pi b^{2}\right)}\left(2 \cos \pi b^{2}\left(L_{2} L_{3}+L_{1} L_{4}\right)+\mathrm{L}_{s}\left(L_{1} L_{3}+L_{2} L_{4}\right)\right) \\
& +\frac{1}{\sqrt{2 \sinh \left(\mathrm{I}_{s} / 2\right)}} e^{+\mathrm{k}_{s} / 2} \frac{\sqrt{c_{12}\left(\mathrm{~L}_{s}\right) c_{34}\left(\mathrm{~L}_{s}\right)}}{2 \sinh \left(\mathrm{I}_{s} / 2\right)} e^{+\mathrm{k}_{s} / 2} \frac{1}{\sqrt{2 \sinh \left(\mathrm{I}_{s} / 2\right)}} \\
& +\frac{1}{\sqrt{2 \sinh \left(\mathrm{I}_{s} / 2\right)}} e^{-\mathrm{k}_{s} / 2} \frac{\sqrt{c_{12}\left(\mathrm{~L}_{s}\right) c_{34}\left(\mathrm{~L}_{s}\right)}}{2 \sinh \left(\mathrm{I}_{s} / 2\right)} e^{-\mathrm{k}_{s} / 2} \frac{1}{\sqrt{2 \sinh \left(\mathrm{I}_{s} / 2\right)}}
\end{aligned}
$$

where $\mathrm{L}_{s}=2 \cosh \left(\mathrm{I}_{s} / 2\right) \equiv \pi_{s}\left(\mathcal{L}_{s}\right)$ and $c_{i j}\left(L_{s}\right)$ was defined in (5.3). The formulae defining the other representations $\pi_{t}$ and $\pi_{u}$ are obtained by simple permutations of indices.

2) The kernel describing the transition between representation $\pi_{s}$ and $\pi_{t}$ is given in terms of the b-6j symbols as

$$
A_{L}^{s t}\left(l_{s}, l_{t}\right)=\left(M\left(\alpha_{t}\right) M\left(\alpha_{s}\right)\right)^{\frac{1}{2}}\left\{\begin{array}{ccc}
\alpha_{1} & \alpha_{2} & \alpha_{s} \\
\alpha_{3} & \alpha_{4} & \alpha_{t}
\end{array}\right\}_{b} \quad \text { if } \quad \alpha_{i}=\frac{Q}{2}+\mathrm{i} \frac{l_{i}}{4 \pi b},
$$

for $i=1,2,3,4, s, t$. The formulae for other pairs of representations are again found by permutations of indices.

The relations between Liouville theory and quantum Teichmüller theory found in [T03] allow one to shortcut the forthcoming self-contained derivation [TeVa] of the claims above. In [T03] it was found in particular that the conformal blocks $\mathcal{F}_{\alpha_{s}}^{(s)}(A \mid Z)$ represent particular wave-functions 
in some representation $\pi_{s}^{\text {Liou, }}$

$$
\psi_{s}\left(l_{s}\right)=\mathcal{F}_{\alpha_{s}}^{(s)}(A \mid Z) \quad \text { if } \quad \alpha_{s}=\frac{Q}{2}+\mathrm{i} \frac{l_{s}}{4 \pi b} .
$$

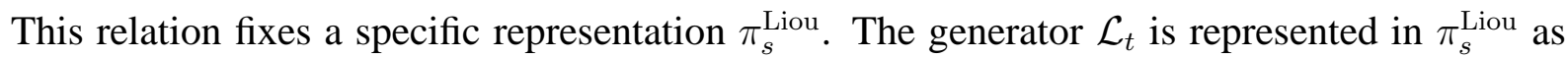
in (5.7) with coefficients $D_{\epsilon}^{\mathrm{Liou}}\left(l_{s}\right)$ that can be extracted from [AGGTV, DGOT] $]^{3}$. Redefining the conformal blocks as in (4.7) is equivalent to a gauge transformation (5.8) which transforms the representation $\pi_{s}^{\text {Liou }}$ to the representation denoted $\pi_{s}^{\text {can }}$. It is straightforward to calculate the coefficients $D_{\epsilon}\left(l_{s}\right)$ from $D_{\epsilon}^{\text {Liou }}\left(l_{s}\right)$ using (4.7) and (4.3). A related observation was recently made in [IOT]. The case of the one-holed torus was discussed along similar lines in [DiGu $]$.

Other normalizations for the b- $6 j$ symbols will correspond to different choices of Darbouxcoordinates. In the normalization used in [DGOT], for example, one would find

$$
\begin{aligned}
\pi_{s}^{\prime}\left(\mathcal{L}_{t}\right)= & \frac{1}{2\left(\cosh I_{s}-\cos 2 \pi b^{2}\right)}\left(2 \cos \pi b^{2}\left(L_{2} L_{3}+L_{1} L_{4}\right)+\mathrm{L}_{s}\left(L_{1} L_{3}+L_{2} L_{4}\right)\right) \\
& +\frac{4}{\sinh \left(\mathrm{I}_{s} / 2\right)} e^{+\mathrm{k}_{s}^{\prime} / 2} \frac{\cosh \frac{\mathrm{I}_{s}+l_{1}-l_{2}}{4} \cosh \frac{\mathrm{I}_{s}+l_{2}-l_{1}}{4} \cosh \frac{\mathrm{I}_{s}+l_{3}-l_{4}}{4} \cosh \frac{\mathrm{I}_{s}+l_{4}-l_{3}}{4}}{\sinh \left(\mathrm{I}_{s} / 2\right)} e^{+\mathrm{k}_{s}^{\prime} / 2} \\
& +\frac{4}{\sinh \left(\mathrm{I}_{s} / 2\right)} e^{-\mathrm{k}_{s}^{\prime} / 2} \frac{\cosh \frac{\mathrm{l}_{s}+l_{1}+l_{2}}{4} \cosh \frac{\mathrm{I}_{s}-l_{1}-l_{2}}{4} \cosh \frac{\mathrm{l}_{s}+l_{3}+l_{4}}{4} \cosh \frac{\mathrm{l}_{s}-l_{3}-l_{4}}{4}}{\sinh \left(\mathrm{I}_{s} / 2\right)} e^{-\mathrm{k}_{s}^{\prime} / 2} .
\end{aligned}
$$

As the analytic properties of the coefficients $D_{\epsilon}\left(l_{s}\right)$ in (5.7) are linked with the analytic properties of the kernels $A_{L}^{s t}\left(l_{s}, l_{t}\right)$ via (5.12), it is no surprise that the kernels $A_{L}^{\prime s t}\left(l_{s}, l_{t}\right)$ associated to the representation $\pi_{s}^{\prime}$ have much better analytic properties than $A_{L}^{s t}\left(l_{s}, l_{t}\right)$ as given by (5.14). One may see see these analytic properties as a profound consequence of the structure of the moduli spaces $\mathcal{M}_{G}(C)$ as algebraic varieties.

\subsection{Classical limit}

The classical counterpart of the expression (5.13b) is found by replacing $l_{s}$ and $k_{s}$ by commuting variables $l_{s}$ and $k_{s}$, respectively, and sending $b \rightarrow 0$. The formulae for the operators $\pi_{s}^{\text {can }}\left(\mathrm{L}_{s}\right)$ and $\pi_{s}^{\text {can }}\left(\mathrm{L}_{t}\right)$ given above are thereby found to be related to the formulae (5.2) for $L_{s}$ and $L_{t}$ in terms of the Darboux coordinates $l_{s}$ and $k_{s}$ for $\mathcal{T}_{0,4}$. We conclude that the representation $\pi_{s}^{\mathrm{can}}$ is the representation associated to the Darboux coordinates discussed in [NRS]. The representation $\pi_{s}^{\prime}$ reproduces (5.5).

Furthermore, by analyzing the classical limit of the relations the relations (5.11) with the help of the saddle-point method one may see that the function $S_{L}^{s t}\left(l_{s}, l_{t}\right)$ which describes the leading semiclassical asymptotics of the kernel $A_{L}^{s t}\left(l_{s}, l_{t}\right)$ via

$$
A_{L}^{s t}\left(l_{s}, l_{t}\right)=\exp \left(\frac{1}{4 \pi \mathrm{i} b^{2}} S_{L}^{s t}\left(l_{s}, l_{t}\right)\right)\left(1+\mathcal{O}\left(b^{2}\right)\right),
$$

\footnotetext{
${ }^{3}$ Our generator $\mathcal{L}_{t}$ corresponds to $2 \cos (\pi b Q) \mathcal{L}\left(\gamma_{2,0}\right)$ in [DGOT].
} 
must coincide with the generating function for the canonical transformation between the Darboux-coordinates $\left(l_{s}, k_{s}\right)$ and $\left(l_{t}, k_{t}\right)$. As this function is known [NRS] to be equal to the volume of the hyperbolic tetrahedron specified by the lengths $\left(l_{1}, l_{2}, l_{3}, l_{4}, l_{s}, l_{t}\right)$, we have found a second proof of the statement that the semiclassical limit of the b- $6 j$ symbols is given by the volume of such tetrahedra.

\section{Applications to supersymmetric gauge theories}

\subsection{Three-dimensional gauge theories on duality walls}

Recently remarkable relations between a certain class $\mathcal{S}$ of $\mathcal{N}=2$ supersymmetric fourdimensional gauge theories and two-dimensional conformal field theories have been discovered in [AGT]. One of the simplest examples for such relations are relations between the partition functions of certain gauge theories on $S^{4}[\overline{\mathrm{Pe}}]$ and physical correlation functions in Liouville theory. The partition function of the $\mathcal{N}=2$ SYM theory with $S U(2)$ gauge group and $N_{f}=4$ hypermultiplets, for example, has a very simple expression in terms of the four-point function (4.1) in Liouville theory. The partition function of the $S$-dual theory would be given by the four-point function (4.2), and the equality between the two expressions [T01] represents a highly nontrivial check of the $S$-duality conjecture.

Interesting generalizations of such relations were recently suggested in [DrGG]: one may consider two four-dimensional theories from class $\mathcal{S}$ on the upper- and lower semispheres of $S^{4}$, respectively, coupled to a three-dimensional theory on the defect $S^{3}$ separating the two semi-spheres. Choosing the two theories to be the $N_{f}=4$ theory and its $S$-dual, for example, the arguments from [DrGG] suggest that the partition function of the full theory should be given by an expression of the form

$$
\int_{(Q / 2+\mathrm{i} \mathbb{R})^{2}} d \alpha_{s} d \alpha_{t}\left(\mathcal{G}_{\alpha_{s}}^{(s)}(A \mid Z)\right)^{*} G_{\alpha_{s} \alpha_{t}}\left[\begin{array}{cc}
\alpha_{3} & \alpha_{2} \\
\alpha_{4} & \alpha_{1}
\end{array}\right] \mathcal{G}_{\alpha_{t}}^{(t)}\left(A \mid Z^{\prime}\right),
$$

using the notations from Section 4. The interpretation in terms of two four-dimensional theories coupled by a defect suggests [DrGG] that the kernel $G_{\alpha_{s} \alpha_{t}}\left[\begin{array}{ll}\alpha_{3} & \alpha_{2} \\ \alpha_{4} & \alpha_{1}\end{array}\right]$ in (6.1) can be interpreted as the partition function of a three-dimensional supersymmetric gauge theory on $S^{3}$ which represents a boundary condition for both of the four-dimensional gauge theories on the semi-spheres of $S^{4}$.

The identification of the three-dimensional gauge theories living on the duality walls may be seen as part of a larger program [TY, DiGu, DiGG] which aims to develop a three-dimensional version of the relations discovered in [AGT]. Roughly speaking, the idea is that there should exist a duality between certain families of three-dimensional supersymmetric gauge theories and 
Chern-Simons theories on suitable three-manifolds. A procedure was described in [DiGG] for the geometric construction of relevant three-dimensional gauge theories from simple building blocks associated to ideal tetrahedra.

In the simpler case where the $N_{f}=4$ theory is replaced by the $\mathcal{N}=4$-supersymmetric gauge theory, an ansatz for the relevant three-dimensional theory was suggested by the work [GW], where this theory was called $T[S U(2)]$. In subsequent work [HLP, HHL2] it was explicit checked that the analog of the kernel $G_{\alpha_{s} \alpha_{t}}$ for this case is given by the partition function of the $T[S U(2)]$ theory. A natural mass-deformation exists for the $T[S U(2)]$-theory, and it was also shown in [HLP, HHL2] that its partition function would essentially coincide with the counterpart of the kernel which would appear in the case of the so-called $\mathcal{N}=2^{*}$-theory rather than the $N_{f}=4$-theory. However, so far no three-dimensional gauge theory which would have the $\mathrm{b}-6 j$ symbols as its partition function has been identified yet.

\subsection{Partition functions of three-dimensional supersymmetric gauge theories}

Let us briefly review the general form of the partition functions for $3 d$ supersymmetric field theories. According to [HHL2], following [KWY, J, HHL1], the partition function for $3 d \mathcal{N}=$ 2 SYM theory with gauge group $G$ and flavor symmetry group $F$ defined on a squashed three sphere has the form

$$
Z(\underline{f})=\int_{-\mathrm{i} \infty}^{\mathrm{i} \infty} \prod_{j=1}^{\operatorname{rank} G} d u_{j} J(\underline{u}) Z^{v e c}(\underline{u}) \prod_{I} Z_{\Phi_{I}}^{c h i r}(\underline{f}, \underline{u}) .
$$

Here $f_{k}$ are the chemical potentials for the flavor symmetry group $F$ while $u_{j}$-variables are associated with the Weyl weights for the Cartan subalgebra of the gauge group $G$. For ChernSimons theories one has $J(\underline{u})=e^{-\pi \mathrm{i} k \sum_{j=1}^{\mathrm{rank} G} u_{j}^{2}}$, where $k$ is the level of CS-term, and for SYM theories one has $J(\underline{u})=e^{2 \pi \mathrm{i} \lambda \sum_{j=1}^{\mathrm{rank} G} u_{j}}$, where $\lambda$ is the Fayet-Illiopoulos term. There are two different contributions to the partition function $\left([6.2): Z^{v e c}(\underline{u})\right.$ which comes from vector superfields and $Z_{\Phi_{I}}^{\text {chir }}(\underline{f}, \underline{u})$ arising from the matter fields. All these terms are expressed in terms of noncompact quantum dilogarithms. The contribution of vector superfield for $G=S U(2)$ which we are interested in coincides with the Plancherel measure (2.16) introduced above,

$$
Z^{v e c}(\underline{u})=M(Q / 2+\mathrm{i} u)
$$

as follows from [HHL2, Equation (5.33)] using (A.15) and (A.16). For each chiral superfield $\Phi_{I}$ the contribution to the partition function is $S_{b}(\alpha)$ where $\alpha$ is some linear combination of the $R$-charge and mass parameters which can be derived from the group representation of the matter content (see, for example, [DSV]$)$. 


\subsection{The b-6j symbols as a partition function}

Although expression (2.28) for b-6 $j$ symbol resembles the partition functions of $3 d \mathrm{SYM}$ theory with $U(1)$ gauge group, it cannot easily be interpreted as partition function for some threedimensional gauge theory since the parameters entering its expression are subject to the condition that their sum equals $2 Q$, while the parameters entering partitions functions are not restricted.

In the course of the derivation of the new formula (2.28) for the b- $6 j$ symbols, as described in Appendix B.2, we have found a few other integral representations for these objects, including

$$
\mathcal{A}_{1} I\left(\begin{array}{ccc}
\frac{Q-\alpha_{t}-\alpha_{1}-\alpha_{4}}{2}+\alpha_{s} & \frac{3 Q-\alpha_{t}-\alpha_{1}-\alpha_{4}}{2}-\alpha_{s} & \frac{Q+\alpha_{1}-\alpha_{4}+\alpha_{t}}{2}-\alpha_{3} \\
\frac{-Q-\alpha_{1}+\alpha_{4}+\alpha_{t}}{2}+\alpha_{2} & \frac{Q-\alpha_{1}+\alpha_{4}+\alpha_{t}}{2}-\alpha_{2} & \frac{-Q+\alpha_{1}-\alpha_{4}+\alpha_{t}}{2}+\alpha_{3}
\end{array}\right),
$$

where we define the integral $I(\underline{\mu})$ as

$$
I(\underline{\mu})=\frac{1}{2} \int_{-\mathrm{i} \infty}^{\mathrm{i} \infty} \frac{\prod_{i=1}^{6} S_{b}\left(\mu_{i} \pm u\right)}{S_{b}( \pm 2 u)} d u, \quad[\underline{\mu}]=\left[\begin{array}{lll}
\mu_{1} & \mu_{2} & \mu_{3} \\
\mu_{4} & \mu_{5} & \mu_{6}
\end{array}\right],
$$

and the prefactor in 6.4 is explicitly given as

$$
\mathcal{A}_{1}=\frac{S_{b}\left(\alpha_{2}+\alpha_{3}-\alpha_{t}\right) S_{b}\left(\alpha_{1}-\alpha_{2}+\alpha_{s}\right) S_{b}\left(-Q+\alpha_{1}+\alpha_{4}+\alpha_{t}\right)}{S_{b}\left(\alpha_{2}+\alpha_{t}-\alpha_{3}\right) S_{b}\left(\alpha_{3}+\alpha_{t}-\alpha_{2}\right) S_{b}\left(\alpha_{3}-\alpha_{4}+\alpha_{s}\right)} .
$$

We would like to point out that this expression, as opposed to (2.28), admits an interpretation as a partition function of the form (6.2) for a certain three-dimensional SYM theory. Namely, the expression (6.4) without coefficient $\mathcal{A}_{1}$ can be interpreted as the partition function of threedimensional $\mathcal{N}=2 \mathrm{SYM}$ theory defined on a squashed three-sphere with $S U(2)$ gauge group and 6 quarks in the fundamental representation of the gauge group. The flavor symmetry group is $S U(6) \times U(1)_{A} \times U(1)_{R}$. The total axial mass is $m_{A}=\frac{1}{6} \sum_{i=1}^{6} \mu_{6}$ while the masses of 6 chiral multiplets then is $m_{i}=\mu_{i}-\frac{1}{6} \sum_{k=1}^{6} \mu_{k}, i=1, \ldots, 6$ (constrained to $\sum_{i=1}^{6} m_{i}=0$ ). We also take the $R$-charge in UV to be 0 . Considering (6.4) as the partition function for $3 d$ $\mathcal{N}=2 \mathrm{SYM}$ theory one obtains a whole series of Seiberg dualities which can be derived from [DSV] by taking $N=1$ there. Keeping in mind the coefficient $\mathcal{A}_{1}$ in (6.4) one sees that the corresponding theory has 8 more singlet chiral fields and the flavor symmetry group is broken to $U(1)^{5} \times U(1)_{A} \times U(1)_{R}$.

We would also like to remark that the identification of the b- $6 j$ symbols as partition functions works straightforwardly only for the b-6j symbol $\left\{\begin{array}{cc|c}\alpha_{1} & \alpha_{2} & \alpha_{s} \\ \alpha_{3} & \alpha_{4} & \alpha_{t}\end{array}\right\}_{b}^{\text {an }}$. The square-roots appearing in the expression for $\left\{\begin{array}{ll|l|l}\alpha_{1} & \alpha_{2} & \alpha_{s} \\ \alpha_{3} & \alpha_{4} & \alpha_{t}\end{array}\right\}_{b}$ seem to prevent a similar interpretation. 


\subsection{Applications to the geometric construction of three-dimensional gauge theories?}

It is interesting to observe that the result for (mass-deformed) $T[S U(2)]$ from [HLP, HHL2], after applications of the same type of identities, can be brought to $3 d \mathcal{N}=2 \mathrm{CS}$ theory with $S U(2)$ gauge group at level 1, 4 quarks and some singlet chiral fields. The above statement can be derived from the following integral identity [SV11]

$$
\begin{aligned}
& \int_{-\mathrm{i} \infty}^{\mathrm{i} \infty} \frac{S_{b}(Q / 4-\mu+m / 2 \pm z)}{S_{b}(3 Q / 4-\mu-m / 2 \pm z)} e^{4 \pi \mathrm{i} \xi z} d z \\
& \quad=\frac{1}{2} e^{2 \pi \mathrm{i}\left(\xi^{2}-\left(\frac{Q}{4}+\frac{m}{2}\right)^{2}+\mu^{2}\right)} S_{b}(Q / 2-m \pm 2 \xi) \int_{-\mathrm{i} \infty}^{\mathrm{i} \infty} \frac{S_{b}\left(\frac{Q}{4}+\frac{m}{2} \pm \mu \pm \xi \pm y\right)}{S_{b}( \pm 2 y)} e^{-2 \pi \mathrm{i} y^{2}} d y .
\end{aligned}
$$

These two observations suggest that there may be an analog of the geometric construction of three-dimensional supersymmetric gauge theories discussed in [DiGG] which is based on building blocks with $S U(2)$ gauge symmetry rather than $U(1)$ gauge symmetry. Indeed, the two three-dimensional partition functions discussed above can be identified with the kernels for the fusion move $A$ and for the modular transformation of the one-punctured torus $S$, respectively. Together with the braiding, the two kernels above generate a representation of the modular groupoid [T08]. This is what one needs to apply standard methods for the combinatorial quantization of Chern-Simons theories to the case of $S L(2, \mathbb{R})$-Chern-Simons theory. It is also suggestive to point out that the number of quarks of the theory whose partition function gives (6.4) nicely matches with the number of angles defining the generic hyperbolic tetrahedron.

We take these observations above as a hint that three-dimensional $\mathcal{N}=2$ SYM theory with $S U(2)$ gauge group and 6 quarks plus some number of singlets could be associated to the nonideal hyperbolic tetrahedron in a future generalization of the constructions in [DiGG], where the triangulations of three-manifold by ideal tetrahedra are replaced by triangulations by nonideal tetrahedra. This raises several interesting questions which should be clarified, including, in particular, the interpretation of normalization changes for $b-6 j$ symbols $(2.18)$ from the point of view of supersymmetric gauge theories.

Acknowledgements We would like to thank T. Dimofte, S. Gukov, R. Kashaev and S. Shatashvili for useful discussions on related topics. 


\section{A. Special functions}

\section{A.1 The function $\Gamma_{b}(x)$}

The function $\Gamma_{b}(x)$ is a close relative of the double Gamma function studied in $[\mathrm{Br}]$. It can be defined by means of the integral representation

$$
\log \Gamma_{b}(x)=\int_{0}^{\infty} \frac{d t}{t}\left(\frac{e^{-x t}-e^{-Q t / 2}}{\left(1-e^{-b t}\right)\left(1-e^{-t / b}\right)}-\frac{(Q-2 x)^{2}}{8 e^{t}}-\frac{Q-2 x}{t}\right)
$$

Important properties of $\Gamma_{b}(x)$ are

$$
\begin{aligned}
\text { functional equation } & \Gamma_{b}(x+b)=\sqrt{2 \pi} b^{b x-\frac{1}{2}} \Gamma^{-1}(b x) \Gamma(x) . \\
\text { analyticity } & \Gamma_{b}(x) \text { is meromorphic, } \\
& \text { poles: } x=-n b-m b^{-1}, n, m \in \mathbb{Z}^{\geq 0} .
\end{aligned}
$$

A useful reference for further properties is [Sp].

\section{A.2 Double Sine function}

The special functions used in this note are all build from the so-called double Sine-function. This function is closely related to the special function here denoted $e_{b}(x)$, which was introduced under the name of quantum dilogarithm in [FK2]. These special functions are simply related to the Barnes double Gamma function $[\overline{\mathrm{Br}}]$, and were also introduced in studies of quantum groups and integrable models in $[\mathrm{F} 2, \mathrm{Ru}, \mathrm{Wo}, \mathrm{V}]$.

In the strip $|\operatorname{Im}(x)|<\frac{Q}{2}$, function $e_{b}(x)$ has the following integral representation

$$
e_{b}(x)=\exp \left\{-\int_{\mathbb{R}+\mathrm{i} 0} \frac{d t}{4 t} \frac{e^{-2 \mathrm{i} t x}}{\sinh b t \sinh \frac{t}{b}}\right\}
$$

where the integration contour goes around the pole $t=0$ in the upper half-plane. The function $s_{b}(x)$ is then related to $e_{b}(x)$ as follows

$$
s_{b}(x)=e^{\frac{\mathrm{i} \pi}{2} x^{2}+\frac{\mathrm{i} \pi}{24}\left(b^{2}+b^{-2}\right)} e_{b}(x)
$$

The analytic continuation of $s_{b}(x)$ to the entire complex plane is a meromorphic function with 
the following properties

$$
\begin{aligned}
\text { functional equation } & \frac{s_{b}\left(x+\frac{\mathrm{i}}{2} b^{ \pm 1}\right)}{s_{b}\left(x-\frac{\mathrm{i}}{2} b^{ \pm 1}\right)}=2 \cosh \left(\pi b^{ \pm 1} x\right), \\
\text { reflection property } & s_{b}(x) s_{b}(-x)=1, \\
\text { complex conjugation } & \frac{s_{b}(x)=s_{b}(-\bar{x}),}{\text { zeros / poles }} \quad\left(s_{b}(x)\right)^{ \pm 1}=0 \Leftrightarrow \pm x \in\left\{\mathrm{i} \frac{Q}{2}+n b+m b^{-1} ; n, m \in \mathbb{Z}^{\geq 0}\right\}, \\
\text { residue } & \operatorname{Res}_{x=-\mathrm{i} \frac{Q}{2}} s_{b}(x)=\frac{\mathrm{i}}{2 \pi}, \\
& s_{b}(x) \sim \begin{cases}e^{-\frac{\mathrm{i} \pi}{2}\left(x^{2}+\frac{1}{12}\left(b^{2}+b^{-2}\right)\right)} & \text { for }|x| \rightarrow \infty,|\arg (x)|<\frac{\pi}{2}, \\
e^{+\frac{\mathrm{i} \pi}{2}\left(x^{2}+\frac{1}{12}\left(b^{2}+b^{-2}\right)\right)} & \text { for }|x| \rightarrow \infty,|\arg (x)|>\frac{\pi}{2} .\end{cases}
\end{aligned}
$$

Of particular importance for us is the behavior for $b \rightarrow 0$, which is given as

$$
e_{b}\left(\frac{v}{2 \pi b}\right)=\exp \left(-\frac{1}{2 \pi b^{2}} \operatorname{Li}_{2}\left(-e^{v}\right)\right)\left(1+\mathcal{O}\left(b^{2}\right)\right) \text {. }
$$

In our paper we mainly use the special function $S_{b}(x)$ defined by

$$
S_{b}(x):=s_{b}\left(\mathrm{i} x-\frac{\mathrm{i}}{2} Q\right)
$$

and has the properties

$$
\begin{aligned}
\qquad \text { self-duality } & S_{b}(x)=S_{b^{-1}}(x), \\
\text { functional equation } & S_{b}\left(x+b^{ \pm 1}\right)=2 \sin \left(\pi b^{ \pm 1} x\right) S_{b}(x), \\
\text { reflection property } & S_{b}(x) S_{b}(Q-x)=1 .
\end{aligned}
$$

The behavior of $S_{b}(x)$ for $b \rightarrow 0$ is then given as

$$
S_{b}\left(\frac{\nu}{2 \pi b}\right)=e^{-\frac{\mathrm{i}}{2 \pi b^{2}}\left(\frac{1}{4} \nu^{2}-\frac{\pi}{2} \nu+\frac{1}{6} \pi^{2}\right)} \exp \left(-\frac{1}{2 \pi \mathrm{i} b^{2}} \operatorname{Li}_{2}\left(e^{\mathrm{i} \nu}\right)\right)\left(1+\mathcal{O}\left(b^{2}\right)\right) .
$$

In terms of $\Gamma_{b}(x)$ the double Sine-function is given as

$$
S_{b}(x)=\frac{\Gamma_{b}(x)}{\Gamma_{b}(Q-x)} .
$$

\section{A.3 The elliptic Gamma function}

The second class of special functions we need here is the elliptic gamma function which appeared implicitly in $[\overline{\mathrm{Bx}}]$ and was introduced in $[\mathrm{Ru}]$

$$
\Gamma(z ; p, q)=\prod_{i, j=0}^{\infty} \frac{1-z^{-1} p^{i+1} q^{j+1}}{1-z p^{i} q^{j}}
$$


satisfying the following properties

$$
\begin{aligned}
\text { symmetry } & \Gamma(z ; p, q)=\Gamma(z ; q, p), \\
\text { functional equations } & \Gamma(q z ; p, q)=\theta(z ; p) \Gamma(z ; p, q), \\
& \Gamma(p z ; p, q)=\theta(z ; q) \Gamma(z ; p, q), \\
\text { reflection property } & \Gamma(z ; p, q) \Gamma\left(\frac{p q}{z} ; p, q\right)=1, \\
\text { zeros } & z \in\left\{p^{i+1} q^{j+1} ; i, j \in \mathbb{Z}^{\geq 0}\right\}, \\
\text { poles } & z \in\left\{p^{-i} q^{-j} ; i, j \in \mathbb{Z}^{\geq 0}\right\}, \\
\text { residue } & \operatorname{Res}_{z=1} \Gamma(z ; p, q)=-\frac{1}{(p ; p)_{\infty}(q ; q)_{\infty}} .
\end{aligned}
$$

Here $\theta(z ; p)$ is a theta-function $\theta(z ; p)=(z ; p)_{\infty}\left(p z^{-1} ; p\right)_{\infty}$.

\section{B. Proof of identity (2.28)}

\section{B.1 The master integral identity}

Let us start from the $V$-function [S03] which is the example from Spiridonov' theory of elliptic hypergeometric integrals $\left[\mathrm{S01}, \mathrm{SO3}_{4}^{4}\right.$ defined by

$$
V(\underline{s})=\kappa \int_{\mathbb{T}} \frac{\prod_{i=1}^{8} \Gamma\left(s_{i} z^{ \pm 1} ; p, q\right)}{\Gamma\left(z^{ \pm 2} ; p, q\right)} \frac{d z}{2 \pi \mathbf{i} z},
$$

where $\prod_{i=1}^{8} s_{i}=(p q)^{2}$ is the so-called balancing condition and

$$
\kappa=\frac{(p ; p)_{\infty}(q ; q)_{\infty}}{2}
$$

with $(z ; q)_{\infty}=\prod_{i=0}^{\infty}\left(1-z q^{i}\right)$. The main building block is the elliptic gamma function defined in (A.18) above.

Theorem 1. [SO3]

$$
V(\underline{s})=\prod_{1 \leq i<j \leq 4} \Gamma\left(s_{i} s_{j} ; p, q\right) \Gamma\left(s_{i+4} s_{j+4} ; p, q\right) V(\underline{t}),
$$

where

$$
t_{i}=\varepsilon s_{i}, i=1,2,3,4 ; \quad t_{i}=\varepsilon^{-1} s_{i}, i=5,6,7,8,
$$

\footnotetext{
${ }^{4}$ From physical point of view this integral is the so-called superconformal index for four-dimensional SQCD theory with $S U(2)$ gauge group and $N_{f}=4$ flavors. The integral transformations for $V$-function describe the multiple duality effect for the above theory [SV10].
} 
and

$$
\varepsilon=\sqrt{\frac{p q}{s_{1} s_{2} s_{3} s_{4}}}=\sqrt{\frac{s_{5} s_{6} s_{7} s_{8}}{p q}} .
$$

The integral identities used in this paper will be obtained from (B.2) by limiting procedures [DS] which reduce the elliptic gamma functions to double Sine functions. First, we reduce $V$ function to the level of hyperbolic $q$-hypergeometric integrals using the reparameterization of variables

$$
z=e^{2 \pi \mathrm{i} r u}, \quad s_{i}=e^{2 \pi \mathrm{i} r \mu_{i}}, \quad i=1, \ldots, 8, \quad p=e^{2 \pi \mathrm{i} b r}, \quad q=e^{2 \pi \mathrm{i} r / b}
$$

and the subsequent limit $r \rightarrow 0$. In this limit the elliptic gamma function has the following asymptotics

$$
\Gamma\left(e^{2 \pi \mathrm{i} r} ; e^{2 \pi \mathrm{i} r b}, e^{2 \pi \mathrm{i} r / b}\right) \underset{r \rightarrow 0}{=} e^{-\pi \mathrm{i}(2 z-b-1 / b) / 12 r} S_{b}(z) .
$$

Using it in the reduction, one obtains an integral lying on the top of a list of integrals emerging as degenerations of the $V$-function (we omit some simple diverging exponential multiplier appearing in this limit together with $-\mathrm{i}$ ),

$$
I_{h}\left(\mu_{1}, \ldots, \mu_{8}\right)=\frac{1}{2} \int_{-\mathrm{i} \infty}^{\mathrm{i} \infty} \frac{\prod_{i=1}^{8} S_{b}\left(\mu_{i} \pm u\right)}{S_{b}( \pm 2 u)} d u
$$

with the balancing condition $\sum_{i=1}^{8} \mu_{i}=2\left(b+b^{-1}\right)$. It has the following symmetry transformation formula descending from the elliptic one

$$
I_{h}\left(\mu_{1}, \ldots, \mu_{8}\right)=\prod_{1 \leq i<j \leq 4} S_{b}\left(\mu_{i}+\mu_{j}\right) \prod_{5 \leq i<j \leq 8} S_{b}\left(\mu_{i}+\mu_{j}\right) I_{h}\left(\nu_{1}, \ldots, \nu_{8}\right)
$$

where $\nu_{i}=\mu_{i}+\xi, \nu_{i+4}=\mu_{i+4}-\xi, i=1,2,3,4$, and the parameter $\xi$ is

$$
2 \xi=\sum_{i=5}^{8} \mu_{i}-b-b^{-1}=b+b^{-1}-\sum_{i=1}^{4} \mu_{i} .
$$

Formula (B.5) will be our main tool in the following.

\section{B.2 Useful corollaries.}

For proving the main transformation formula which allows us to get from (2.17) the expression (2.28) we need following corollaries.

\section{Corollary 1.}

$$
I(\underline{\mu})=S_{b}\left(\mu_{5}+\mu_{6}\right) S_{b}\left(2 Q-\sum_{i=1}^{6} \mu_{6}\right) \prod_{1 \leq i<j \leq 4} S_{b}\left(\mu_{i}+\mu_{j}\right) I(\underline{\nu}),
$$


where we define the integral $I(\underline{\mu})$ as

$$
I(\underline{\mu})=\frac{1}{2} \int_{-\mathrm{i} \infty}^{\mathrm{i} \infty} \frac{\prod_{i=1}^{6} S_{b}\left(\mu_{i} \pm u\right)}{S_{b}( \pm 2 u)} d u .
$$

Here we have

$$
\left[\nu_{1}, \nu_{2}, \nu_{3}, \nu_{4}, \nu_{5}, \nu_{6}\right]=\left[\mu_{1}+\xi, \mu_{2}+\xi, \mu_{3}+\xi, \mu_{4}+\xi, \mu_{5}-\xi, \mu_{6}-\xi\right]
$$

and

$$
2 \xi=Q-\sum_{i=1}^{4} \mu_{i}
$$

Later it will be convenient to write 6 variables $\underline{\mu}$ in the following way

$$
[\underline{\mu}]=\left[\begin{array}{lll}
\mu_{1} & \mu_{2} & \mu_{3} \\
\mu_{4} & \mu_{5} & \mu_{6}
\end{array}\right]
$$

\section{Corollary 2. :}

$$
J(\underline{\mu}, \underline{\nu})=\prod_{i=1}^{3} S_{b}\left(\mu_{i}+\nu_{4}\right) S_{b}\left(\nu_{i}+\mu_{4}\right) I(\underline{\rho})
$$

with

$$
J(\underline{\mu}, \underline{\nu})=\int_{-\mathrm{i} \infty}^{\mathrm{i} \infty} \prod_{i=1}^{4} S_{b}\left(\mu_{i}-u\right) S_{b}\left(\nu_{i}+u\right) d u,
$$

which has $U(1)$ gauge symmetry, and the balancing condition $\sum_{i=1}^{4}\left(\mu_{i}+\nu_{i}\right)=2 Q$. Here we have

$$
\left[\rho_{1}, \rho_{2}, \rho_{3}, \rho_{4}, \rho_{5}, \rho_{6}\right]=\left[\mu_{1}+\xi, \mu_{2}+\xi, \mu_{3}+\xi, \nu_{1}-\xi, \nu_{2}-\xi, \nu_{3}-\xi\right]
$$

and

$$
2 \xi=Q-\nu_{4}-\sum_{i=1}^{3} \mu_{i}=-Q+\mu_{4}+\sum_{i=1}^{3} \nu_{i} .
$$

Again it is useful to have the following notation

$$
[\underline{\mu}, \underline{\nu}]=\left[\begin{array}{cccc}
\mu_{1} & \mu_{2} & \mu_{3} & \mu_{4} \\
\nu_{1} & \nu_{2} & \nu_{3} & \nu_{4}
\end{array}\right],
$$

The inversion of Corollary 2 is the following

\section{Corollary 3.}

$$
I(\underline{\rho})=\prod_{1 \leq i<j \leq 3} S_{b}\left(\rho_{i}+\rho_{j}\right) S_{b}\left(\rho_{i+3}+\rho_{j+3}\right) J(\underline{\mu}, \underline{\nu}),
$$


and the balancing condition $\sum_{i=1}^{4} \mu_{i}+\nu_{i}=2 Q$. Here we have

$$
\left[\begin{array}{cccc}
\mu_{1} & \mu_{2} & \mu_{3} & \mu_{4} \\
\nu_{1} & \nu_{2} & \nu_{3} & \nu_{4}
\end{array}\right]=\left[\begin{array}{cccc}
\rho_{1}-x & \rho_{2}-x & \rho_{3}-x & Q-\rho_{456}-x \\
\rho_{4}+x & \rho_{5}+x & \rho_{6}+x & Q-\rho_{123}+x
\end{array}\right],
$$

where $\rho_{123}=\rho_{1}+\rho_{2}+\rho_{3}, \rho_{456}=\rho_{4}+\rho_{5}+\rho_{6}$, and $x$ is arbitrary.

Corollary 4. :

$$
I(\underline{\mu})=S_{b}\left(2 Q-\sum_{i=1}^{6} \mu_{i}\right) \prod_{1 \leq i<j \leq 6} S_{b}\left(\mu_{i}+\mu_{j}\right) I(Q / 2-\underline{\mu}) .
$$

To get the desired transformation formulas one should use the following asymptotic formulas when some of the parameters go to infinity

$$
\begin{aligned}
& \lim _{u \rightarrow \infty} e^{\frac{\pi \mathrm{i}}{2} B_{2,2}(u)} S_{b}(u)=1, \quad \text { for } \arg b<\arg u<\arg 1 / b+\pi, \\
& \lim _{u \rightarrow \infty} e^{-\frac{\pi \mathrm{i}}{2} B_{2,2}(u)} S_{b}(u)=1, \quad \text { for } \arg b-\pi<\arg u<\arg 1 / b .
\end{aligned}
$$

By taking different restrictions for the parameters one can get lots of identities from the integral identity (B.5). Let us take

$$
\mu_{1} \rightarrow \mu_{1}+\mu ; \quad \mu_{5} \rightarrow \mu_{5}-\mu
$$

with the following limit $\mu \rightarrow \infty$. The left hand-side of (B.5) gives

$$
I_{h}\left(\mu_{2}, \mu_{3}, \mu_{4}, \mu_{6}, \mu_{7}, \mu_{8}\right)=\frac{1}{2} \int_{-\mathrm{i} \infty}^{\mathrm{i} \infty} \frac{\prod_{i=2}^{4} S_{b}\left(\mu_{i} \pm z\right) S_{b}\left(\mu_{i+4} \pm z\right)}{S_{b}( \pm 2 z)} d z,
$$

without any restrictions for parameters $\mu_{2}, \mu_{3}, \mu_{4}, \mu_{6}, \mu_{7}, \mu_{8}$ and in the right hand-side one needs to shift the integration variable $z \rightarrow z-\mu / 2$ and afterwards taking the limit $\mu \rightarrow \infty$ which gives

$$
\begin{aligned}
\prod_{2 \leq i<j \leq 4} S_{b}\left(\mu_{i}+\mu_{j}\right) S_{b}\left(\mu_{i+4}+\mu_{j+4}\right) \\
\times \int_{-\mathrm{i} \infty}^{\mathrm{i} \infty} d z S_{b}\left(\xi+z+\left(\mu_{1}+\mu_{5}\right) / 2\right) S_{b}\left(\left(\mu_{1}+\mu_{5}\right) / 2-\xi-z\right) \\
\quad \times \prod_{i=2}^{4} S_{b}\left(\mu_{i}+\xi-\left(\mu_{1}+\mu_{5}\right) / 2-z\right) S_{b}\left(\mu_{i+4}-\xi+\left(\mu_{1}+\mu_{5}\right) / 2+z\right) d z
\end{aligned}
$$

and $2 \xi=Q-\sum_{i=2}^{4} \mu_{i}$.

Inverting now the equality $(\overline{\mathrm{B} .12})=(\overline{\mathrm{B} .13})$ one gets Corollary 3 , To get Corollary 1 one takes the limit $\mu_{7}, \mu_{8} \rightarrow \infty$ such that $\mu_{7}-\mu_{8}=O(1)$ in (B.5). 
Application of (B.2) twice and thrice gives new integral transformations formulas for (B.1) while further application of (B.2) does not lead to new integral transformations. It can be shown [S08]

$$
V\left(s_{1}, \ldots, s_{8}\right)=\prod_{1 \leq i<j \leq 8} \Gamma\left(s_{i} s_{j} ; p, q\right) V\left(\frac{\sqrt{p q}}{s_{1}}, \ldots, \frac{\sqrt{p q}}{s_{8}}\right),
$$

the reduction to the hyperbolic level of which brings to Corollary 4

In [SV11] other reductions of $V$-functions were considered in connections with the so-called state integral for $\mathbf{4}_{1}$ knot [Hi1] and with the kernel of $S$-move [T03].

\section{B.3 Derivation of the indentity (2.28)}

Let us start from the expression (2.17) and apply Corollary 2 taking parameters as

$$
[\underline{\mu}, \underline{\nu}]=\left[\begin{array}{ccc}
Q \pm\left(\alpha_{s}-\frac{Q}{2}\right) & \alpha_{2}+\alpha_{4}+\alpha_{t}-\frac{Q}{2} & \alpha_{2}+\alpha_{4}-\alpha_{t}+\frac{Q}{2} \\
-\alpha_{4} \pm\left(\alpha_{3}-\frac{Q}{2}\right) & \frac{Q}{2}-\alpha_{1}-\alpha_{2} & -\frac{Q}{2}+\alpha_{1}-\alpha_{2}
\end{array}\right],
$$

one gets

$$
\mathcal{A}_{1} I\left(\begin{array}{ccc}
\frac{Q-\alpha_{t}-\alpha_{1}-\alpha_{4}}{2}+\alpha_{s} & \frac{3 Q-\alpha_{t}-\alpha_{1}-\alpha_{4}}{2}-\alpha_{s} & \frac{Q+\alpha_{1}-\alpha_{4}+\alpha_{t}}{2}-\alpha_{3} \\
\frac{-Q-\alpha_{1}+\alpha_{4}+\alpha_{t}}{2}+\alpha_{2} & \frac{Q-\alpha_{1}+\alpha_{4}+\alpha_{t}}{2}-\alpha_{2} & \frac{-Q+\alpha_{1}-\alpha_{4}+\alpha_{t}}{2}+\alpha_{3}
\end{array}\right)
$$

with

$$
\mathcal{A}_{1}=\frac{S_{b}\left(\alpha_{2}+\alpha_{3}-\alpha_{t}\right) S_{b}\left(\alpha_{1}-\alpha_{2}+\alpha_{s}\right) S_{b}\left(-Q+\alpha_{1}+\alpha_{4}+\alpha_{t}\right)}{S_{b}\left( \pm\left(Q-2 \alpha_{t}\right)\right) S_{b}\left(\alpha_{2}+\alpha_{t}-\alpha_{3}\right) S_{b}\left(\alpha_{3}+\alpha_{t}-\alpha_{2}\right) S_{b}\left(\alpha_{3}-\alpha_{4}+\alpha_{s}\right)} .
$$

The integral in $(\mathrm{B} .15)$ is defined for $\alpha_{k} \in Q / 2+\mathrm{i} \mathbb{R}$ by using a contour $\widetilde{C}$ that approaches $\frac{Q}{4}+\mathrm{i} \mathbb{R}$ near infinity, and passes the real axis in $\left(-\frac{Q}{4}, \frac{Q}{4}\right)$, and for other values of $\alpha_{k} \in \frac{Q}{2}+\mathrm{i} \mathbb{R}$ by analytic continuation.

Applying Corollary 1 to (B.15) (with the order of parameters as staying in $(\mathrm{B} .15)$ ) one obtains

$$
\mathcal{A}_{2} I\left(\begin{array}{ccc}
\alpha_{s}+\frac{\alpha_{3}-\alpha_{2}-\alpha_{t}}{2} & Q-\alpha_{s}+\frac{\alpha_{3}-\alpha_{2}-\alpha_{t}}{2} & \alpha_{1}+\frac{\alpha_{t}-\alpha_{2}-\alpha_{3}}{2} \\
\alpha_{4}-Q+\frac{\alpha_{2}+\alpha_{3}+\alpha_{t}}{2} & Q-\alpha_{1}+\frac{\alpha_{t}-\alpha_{2}-\alpha_{3}}{2} & -\alpha_{4}+\frac{\alpha_{2}+\alpha_{3}+\alpha_{t}}{2}
\end{array}\right),
$$

defined by the contour $\widetilde{C}$ and where

$$
\mathcal{A}_{2}=\frac{S_{b}\left(\alpha_{2}+\alpha_{3}-\alpha_{t}\right) S_{b}\left(-\alpha_{1}+\alpha_{2}+\alpha_{s}\right) S_{b}\left(\alpha_{1}+\alpha_{4}-\alpha_{t}\right) S_{b}\left(2 Q-\alpha_{3}-\alpha_{4}-\alpha_{s}\right)}{S_{b}\left( \pm\left(Q-2 \alpha_{t}\right)\right) S_{b}\left(\alpha_{3}-\alpha_{4}+\alpha_{s}\right) S_{b}\left(\alpha_{3}+\alpha_{4}-\alpha_{s}\right)} .
$$

On the next step we apply Corollary 4 to $(\underline{B .16})$ and get

$$
\mathcal{A}_{3} I\left(\begin{array}{ccc}
-\alpha_{s}+\frac{Q+\alpha_{2}-\alpha_{3}+\alpha_{t}}{2} & \alpha_{s}+\frac{-Q+\alpha_{2}-\alpha_{3}+\alpha_{t}}{2} & -\alpha_{1}+\frac{Q+\alpha_{2}+\alpha_{3}-\alpha_{t}}{2} \\
-\alpha_{4}+\frac{3 Q-\alpha_{2}-\alpha_{3}-\alpha_{t}}{2} & \alpha_{1}+\frac{-Q+\alpha_{2}+\alpha_{3}-\alpha_{t}}{2} & \alpha_{4}+\frac{Q-\alpha_{2}-\alpha_{3}-\alpha_{t}}{2}
\end{array}\right),
$$


with the same contour $\widetilde{C}$ and

$$
\begin{aligned}
\mathcal{A}_{3}= & \frac{S_{b}\left(\alpha_{1}-\alpha_{2}+\alpha_{s}\right) S_{b}\left(\alpha_{1}-\alpha_{4}+\alpha_{t}\right) S_{b}\left(\alpha_{1}+\alpha_{4}-\alpha_{t}\right)}{S_{b}\left( \pm\left(Q-2 \alpha_{t}\right)\right) S_{b}\left(\alpha_{2}-\alpha_{3}+\alpha_{t}\right) S_{b}\left(\alpha_{1}+\alpha_{2}-\alpha_{s}\right) S_{b}\left(2 Q-\alpha_{1}-\alpha_{4}-\alpha_{t}\right)} \\
& \times \frac{S_{b}\left(2 Q-\alpha_{1}-\alpha_{2}-\alpha_{s}\right) S_{b}\left(-\alpha_{1}+\alpha_{4}+\alpha_{t}\right)}{S_{b}\left(-\alpha_{3}+\alpha_{4}+\alpha_{s}\right) S_{b}\left(2 Q-\alpha_{2}-\alpha_{3}-\alpha_{t}\right) S_{b}\left(\alpha_{1}+\alpha_{4}-\alpha_{t}\right) S_{b}\left(-\alpha_{2}+\alpha_{3}+\alpha_{t}\right)} .
\end{aligned}
$$

Finally, we apply Corollary 3 for (B.17) with slightly permuted parameters (since the integral has $S_{6}$ permutation symmetry over parameters)

$$
\mathcal{A}_{3} I\left(\begin{array}{ccc}
\alpha_{s}+\frac{-Q+\alpha_{2}-\alpha_{3}+\alpha_{t}}{2} & \alpha_{1}+\frac{-Q+\alpha_{2}+\alpha_{3}-\alpha_{t}}{2} & -\alpha_{4}+\frac{3 Q-\alpha_{2}-\alpha_{3}-\alpha_{t}}{2} \\
-\alpha_{s}+\frac{Q+\alpha_{2}-\alpha_{3}+\alpha_{t}}{2} & -\alpha_{1}+\frac{Q+\alpha_{2}+\alpha_{3}-\alpha_{t}}{2} & \alpha_{4}+\frac{Q-\alpha_{2}-\alpha_{3}-\alpha_{t}}{2}
\end{array}\right),
$$

together with taking

$$
x=-\frac{Q+\alpha_{2}+\alpha_{3}+\alpha_{t}}{2}-\alpha_{4}
$$

to get (2.28) which proves the identity (2.28) in the main part of the text.

\section{References}

[AGT] L. F. Alday, D. Gaiotto, and Y. Tachikawa, Liouville Correlation Functions from Fourdimensional Gauge Theories, Lett. Math. Phys. 91 (2010) 167-197.

[AGGTV] L. F. Alday, D. Gaiotto, S. Gukov, Y. Tachikawa, H. Verlinde, Loop and surface operators in $\mathcal{N}=2$ gauge theory and Liouville modular geometry, J. High Energy Phys. 1001 (2010) 113.

[ALPS] D. Alessandrini, L. Liu. A. Papadopoulos, W. Su, The behaviour of Fenchel-Nielsen distance under a change of pants decomposition, arXiv:1105.0202.

[AK] J. E. Andersen, R. Kashaev, A TQFT from quantum Teichmüller theory, arXiv:1109.6295,

[BMS1] V. V. Bazhanov, V. V. Mangazeev, S. M. Sergeev, Faddeev-Volkov solution of the Yang-Baxter equation and discrete conformal symmetry, Nucl. Phys. B 784 (2007) 234-258.

[BMS2] V. V. Bazhanov, V. V. Mangazeev, S. M. Sergeev, Quantum geometry of 3-dimensional lattices, J. Stat. Mech. 0807 (2008) P07004.

[Br] E.W. Barnes: Theory of the double gamma function, Phil. Trans. Roy. Soc. A196 (1901) 265-388.

[Bx] R. J. Baxter, Partition function of the eight-vertex lattice model, Ann. Phys. (NY) 70 (1972) 193228.

[BT1] A. G. Bytsko and J. Teschner, R-operator, co-product and Haar-measure for the modular double of $U_{q}(\mathfrak{s l}(2, \mathbb{R}))$, Comm. Math. Phys. 240 (2003) 171-196.

[BT2] A. G. Bytsko and J. Teschner, Quantization of models with non-compact quantum group symmetry: Modular XXZ magnet and lattice sinh-Gordon model, J. Phys. A A 39 (2006) 12927-12981.

[CF99] V. V. Fock, L. O. Chekhov, Quantum Teichmüller spaces, Theor. and Math. Phys. 120 (1999) $1245-1259$. 
[CF00] V. V. Fock, L. O. Chekhov, Observables in $3 D$ gravity and geodesic algebras, Czechoslovak J. Phys. 50 (2000) 1201-1208.

[DS] J. F. van Diejen and V. P. Spiridonov, Unit circle elliptic beta integrals, Ramanujan J. 10 (2005) 187-204.

[DGLZ] T. Dimofte, S. Gukov, J. Lenells, Don Zagier, Exact Results for Perturbative Chern-Simons Theory with Complex Gauge Group, Commun. Num. Theor. Phys. 3 (2009) 363-443.

[DiGu] T. Dimofte, S. Gukov, Chern-Simons Theory and S-duality, arXiv:1106.4550 [hep-th].

[DiGG] T. Dimofte, D. Gaiotto and S. Gukov, Gauge Theories Labelled by Three-Manifolds, arXiv:1108.4389 [hep-th].

[DSV] F. A. H. Dolan, V. P. Spiridonov and G. S. Vartanov, From $4 d$ superconformal indices to $3 d$ partition functions, Phys. Lett. B 704 (2011) 234-241.

[DrGG] N. Drukker, D. Gaiotto, and J. Gomis, The Virtue of Defects in $4 D$ Gauge Theories and $2 D$ CFTs, J. High Energy Phys. 1106 (2011) 025.

[DGOT] N. Drukker, J. Gomis, T. Okuda, J. Teschner, Gauge Theory Loop Operators and Liouville Theory, J. High Energy Phys. 1002 (2010) 057.

[F2] L. D. Faddeev: Discrete Heisenberg-Weyl group and modular group, Lett. Math. Phys. 34 (1995) 249-254.

[FK2] L.D. Faddeev and R. M. Kashaev: Quantum dilogarithm, Mod. Phys. Lett. A9 (1994) 427-434.

[F99] L. D. Faddeev, Modular double of a quantum group, Confrence Mosh Flato 1999, Vol. I (Dijon), 149-156, Math. Phys. Stud., 21, Kluwer Acad. Publ., Dordrecht, 2000, arXiv:math/9912078

[Fo97] V. Fock, Dual Teichmüller spaces, arXiv:dg-ga/9702018.

[GW] D. Gaiotto and E. Witten, $S$-Duality of Boundary Conditions In $\mathcal{N}=4$ Super Yang-Mills Theory, arXiv:0807.3720 [hep-th].

[HHL1] N. Hama, K. Hosomichi, and S. Lee, Notes on SUSY gauge theories on three-sphere, J. High Energy Phys. 1103 (2011) 127.

[HHL2] N. Hama, K. Hosomichi, and S. Lee, SUSY gauge theories on squashed three-spheres, J. High Energy Phys. 1105 (2011) 014.

[Hi1] K. Hikami, Hyperbolic Structure Arising from a Knot Invariant, Int. J. Mod. Phys. A16 (2001) 3309-3333.

[Hi2] K. Hikami, Hyperbolicity of Partition Function and Quantum Gravity, Nucl. Phys. B616 (2001) 537-548.

[Hi3] K. Hikami, Generalized Volume Conjecture and the A-Polynomials - the Neumann-Zagier Potential Function as a Classical Limit of Quantum Invariant, J. Geom. Phys. 57 (2007) 1895-1940.

[HLP] K. Hosomichi, S. Lee, and J. Park, AGT on the S-duality Wall, J. High Energy Phys. 1012 (2010) 079.

[Ip] I. C. H. Ip, Representation of the Quantum Plane, its Quantum Double, and Harmonic Analysis on $G L_{q}^{+}(2, R)$, arXiv:1108.5365. 
[IOT] Y. Ito, T. Okuda, M. Taki, Line operators on $S^{1} \times R^{3}$ and quantization of the Hitchin moduli space, arXiv:1111.4221.

[J] D. L. Jafferis, The Exact Superconformal R-Symmetry Extremizes Z, arXiv:1012.3210,

[KWY] A. Kapustin, B. Willett, and I. Yaakov, Exact results for Wilson loops in superconformal ChernSimons theories with matter, J. High Energy Phys. 1003 (2010) 089.

[K] R. M. Kashaev, The hyperbolic volume of knots from the quantum dilogarithm, Lett. Math. Phys. 39 (1997) 269-275.

[Ka98] R. M. Kashaev, Quantization of Teichmüller spaces and the quantum dilogarithm, Lett. Math. Phys. 43 (1998), no. 2, 105-115.

[K2] R. Kashaev, The quantum dilogarithm and Dehn twists in quantum Teichmller theory, "Integrable structures of exactly solvable two-dimensional models of quantum field theory", (Kiev, 2000) 211221, NATO Sci. Ser. II Math. Phys. Chem., 35, Kluwer Acad. Publ., Dordrecht, 2001.

[MS] G. Moore, N. Seiberg, Classical and quantum conformal field theory, Comm. Math. Phys. 123 (1989) 177-254

[MT] S. Mizoguchi and T. Tada, Three-dimensional gravity from the Turaev-Viro invariant, Phys. Rev. Lett. 68 (1992) 1795-1798.

[MY] J. Murakami and M. Yano, On the volume of a hyperbolic and spherical tetrahedron, Comm. Anal. Geom. 13 (2005), no. 2, 379-400.

[N] N. A. Nekrasov, Seiberg-Witten prepotential from instanton counting, Adv. Theor. Math. Phys. 7 (2003) 831-864.

[NRS] N. Nekrasov, A. Rosly, S. Shatashvili, Darboux coordinates, Yang-Yang functional, and gauge theory, Nucl. Phys. Proc. Suppl. 216 (2011) 69-93.

[Pe] V. Pestun, Localization of gauge theory on a four-sphere and supersymmetric Wilson loops, arXiv:0712.2824 [hep-th].

[PR] G. Ponzano, T. Regge, Semiclassical limit of Racah coefficients, "Spectroscopic and Group Theoretical Methods in Physics", ed. F. Bloch (North-Holland, Amsterdam, 1968).

[PT1] B. Ponsot and J. Teschner, Liouville bootstrap via harmonic analysis on a noncompact quantum group, arXiv:hep-th/9911110.

[PT2] B. Ponsot and J. Teschner, Clebsch-Gordan and Racah-Wigner coefficients for a continuous series of representations of $U_{q}(s l(2, \mathbb{R}))$, Commun. Math. Phys. 224 (2001) 613-655.

[Ru] S. N. M. Ruijsenaars: First order analytic difference equations and integrable quantum systems, J. Math. Phys. 38 (1997) 1069-1146.

[S01] V.P. Spiridonov, On the elliptic beta function, Uspekhi Mat. Nauk 56 (1) (2001) 181-182 (Russian Math. Surveys 56 (1) (2001) 185-186).

[S03] V. P. Spiridonov, Theta hypergeometric integrals, Algebra i Analiz 15 (6) (2003) 161-215 (St. Petersburg Math. J. 15 (6) (2004), 929-967).

[S08] V. P. Spiridonov, Essays on the theory of elliptic hypergeometric functions, Uspekhi Mat. Nauk 63 (3) (2008), 3-72 (Russian Math. Surveys 63 (3) (2008), 405-472). 
[SV10] V. P. Spiridonov and G. S. Vartanov, Superconformal indices for $\mathcal{N}=1$ theories with multiple duals, Nucl. Phys. B 824 (2010) 192-216.

[SV11] V. P. Spiridonov and G. S. Vartanov, Elliptic hypergeometry of supersymmetric dualities II. Orthogonal groups, knots, and vortices, arXiv:1107.5788 [hep-th].

[Sp] M. Spreafico, On the Barnes double zeta and Gamma functions, Journal of Number Theory 129 (2009) 2035-2063.

[TY] Y. Terashima and M. Yamazaki, $S L(2, \mathbb{R})$ Chern-Simons, Liouville, and Gauge Theory on Duality Walls, J. High Energy Phys. 1108 (2011) 135.

[T01] J. Teschner, Liouville theory revisited, Class. Quant. Grav. 18 (2001) R153-R222.

[T03] J. Teschner, On the relation between quantum Liouville theory and the quantized Teichmüller spaces, Int. J. Mod. Phys. A19S2 (2004), 459-477; From Liouville theory to the quantum geometry of Riemann surfaces, Cont. Math. 437 (2007) 231-246.

[T05] J. Teschner, An analog of a modular functor from quantized Teichmüller theory, "Handbook of Teichmüller theory", (A. Papadopoulos, ed.) Volume I, EMS Publishing House, Zürich 2007, 685760.

[T08] J. Teschner, Nonrational conformal field theory, "New Trends in Mathematical Physics" (Selected contributions of the XVth ICMP), Vladas Sidoravicius (ed.), Springer Science and Business Media B.V. 2009.

[TeVa] J. Teschner, G. S. Vartanov, In preparation.

[TuVi] V. G. Turaev, O. Y. Viro, State Sum Invariants of 3-Manifolds and Quantum 6j-Symbols, Topology 31 (1992) 865-902.

[V] A. Yu. Volkov: Noncommutative hypergeometry, Commun. Math. Phys. 258 (2005) 257-273.

[Wo] S. L. Woronowicz: Quantum exponential function, Rev. Math. Phys. 12 (2000) 873-920. 